\title{
THE ALEXANDER POLYNOMIAL OF A CONFIGURATION OF SKEW LINES IN 3-SPACE
}

\begin{abstract}
Rudi Penne
In Penne, 1995, we observed that the multi-variable Alexander polynomial can be computed by means of "multi-variable Burau matrices", the entries of which can be visualized by a "path model". In this article we introduce the Alexander polynomial for line configurations. The correspondence between "adjacency of lines" in a configuration, and the factorization of its Alexander polynomial can be well understood by way of the path model.
\end{abstract}

\section{Introduction.}

In a previous paper $([\mathbf{1 2}])$ we introduced a new machinery to compute the multi-variable Alexander polynomial $\Delta_{L}$ of a link $L$ with $k$ components. Although everything was stated for pure links, the techniques of [12] apply for general links if one makes a small additional observation (Theorem 1).

Let us quickly repeat the basic facts of the (non-reduced) Alexander polynomial. If we regard the link $L$ as a closed braid $\bar{\beta}, \beta \in B_{n}$, and if we use Artin's representation of the braid group as free automorphisms,

$$
\xi: B_{n} \rightarrow \operatorname{Aut}\left(F_{n}\right),
$$

we can present the group of $L, G(L)$ (the fundamental group of its complement), by $n$ generators $x_{1}, \ldots, x_{n}$ subject to the relations

$$
x_{i}=\xi(\beta)\left(x_{i}\right), \quad 1 \leq i \leq n .
$$

For more details, we refer to $[2,3]$. It is well known that each of the $n$ relations in (2) is implied by the others, allowing us to drop one relation, and we come up with a group presentation for $G(L)$ of "deficiency 1" (one less relation than generators):

$$
G(L)=\left(x_{1}, \ldots, x_{n}: r_{1}, \ldots, r_{n-1}\right) .
$$

With the aid of free differential calculus in the group $\operatorname{ring} \mathbf{Z}\left[F_{n}\right]$, as introduced by R.H. Fox in [7], we can construct the Jacobian of $G(L)$ :

$$
\left(\frac{\partial r_{i}}{\partial x_{j}}\right)_{i, j}^{\phi}
$$


where the quotient map $\phi: F_{n} \rightarrow G(L)$ is extended to go from $\mathbf{Z}\left[F_{n}\right]$ to $\mathbf{Z}[G(L)]$, acting entry-wise on the matrix. Finally, if we consider the abelianizer from $G(L)$ to $H=G(L) /[G(L), G(L)]$, and extend it to the corresponding group rings, it can be applied to the entries of the Jacobian, resulting in an Alexander matrix $A(L)$ for $L$. We refer to [8] for more details. One can show that the abelianization $H$ of the link group $G(L)$ is torsion free with rank equal to $k$, the number of components of $L$. So, $A(L)$ has its entries in the ring $\mathbf{Z}\left[t_{1}^{ \pm 1}, \ldots, t_{k}^{ \pm 1}\right]$. This matrix highly depends on the chosen presentation for $G(L)$. However, the ideal $I$ generated by the maximal minors of $A(L)$ is a group invariant, and hence a link invariant. In the case $k=1, L$ is a knot and $I$ is a principal ideal, whose generator is exactly the classical Alexander polynomial $\Delta_{L}(t)([\mathbf{1}])$. If $k \geq 2, I$ is the product of a principal ideal $I_{0}$ and the augmentation ideal, motivating R.H. Fox to define the generator of $I_{0}$ as the multi-variable Alexander polynomial $\Delta_{L}\left(t_{1}, \ldots, t_{k}\right)$ of $L$. In any case, $\Delta_{L}$ is only defined up to multiplicative units in $\mathbf{Z}[H]$.

Let now $A(\beta)$ denote the $n \times n$ matrix whose $i$-th row consists of the free partial derivatives of $\xi(\beta)\left(x_{i}\right)$, canonically mapped to $\mathbf{Z}[H]$. That is,

$$
A(\beta)_{i j}=a\left(\frac{\partial}{\partial x_{j}} \xi(\beta)\left(x_{i}\right)\right),
$$

where $a: \mathbf{Z}\left[F_{n}\right] \rightarrow \mathbf{Z}[H]$ composes the quotient map with the abelianizer. Notice that

$$
\left(A(\beta)-I_{n}\right)_{i j}=a\left(\frac{\partial}{\partial x_{j}} \xi(\beta)\left(x_{i}\right) \cdot x_{i}^{-1}\right),
$$

such that an Alexander matrix $A(L)$ for $L$ is obtained by deleting any row of $A(\beta)-I_{n}([3])$. We conclude that the Alexander polynomial of a link $L=\bar{\beta}$ is immediately obtained once we have computed $A(\beta)$. In this article, $A(\beta)$ will be computed by associating so-called multi-variable Burau matrices with the crossings of $\beta$, multiplying them in order of appearance. This generalizes the well-known procedure for knots $(k=1)$, where $A(\beta)$ is obtained as the Burau representation $\tau_{B}(\beta)$ of $\beta$.

The fundamental properties of the multi-variable Burau matrices can be found in [12]. In this article we focus on some of their applications, showing how they can improve our understanding of the Alexander polynomial. As pointed out in [12], the entries of a multi-variable Burau matrix can be easily visualized by considering topological paths on the involved braid diagram, which are evaluated by appropriate weights. This path model will be explained in Section 4. The main goal of this article is to illustrate the elegance of the path model by obtaining fundamental combinatorial proofs for classical theorems of Morton, Torres and Sumners-Woods on the Alexander polynomial (Section 5 and Section 6). 
Most of our material will be stated in terms of a special class of links. More precisely, we are motivated by the isotopy problem of line configurations, that are configurations of a finite number of lines in $\mathbf{R P}^{3}$. Two line configurations are called rigidly isotopic if there exists an ambient isotopy that connects them and during which the geometric lines are maintained. We will moreover always assume that the lines are pairwise disjoint (nonsingular configurations). If we pull back such configurations to the covering space $S^{3}$ of $\mathbf{R P}^{3}$, we come up with mutually linked circles. Links which are obtained in this way are called Klein links. The classification of Klein links is an interesting variation of the general theme in Knot Theory, while on the other hand it provides a natural relaxation for the rigid isotopy classification of line configurations.

In the Appendix we list the multi-variable Alexander polynomials for all non-singular line configurations of at most six lines (more precisely, we present the Alexander polynomials for their Klein links). In [16], O.Ya. Viro introduced the concept of adjacency for line configurations. Two lines in a configuration are called adjacent, if they can be brought "arbitrarily close to each other" by a rigid isotopy of the entire configuration. The adjacency relation in a line configuration corresponds to "simple factors" of its Alexander polynomial, which is very striking in the list of the Appendix. This correspondence is elaborated in Section 6 .

\section{Multi-variable Burau matrices.}

In this section we explain our technique for computing the non-reduced (= multi-variable) Alexander polynomial $\Delta_{L}\left(t_{1}, \ldots, t_{k}\right)$ of a link $L$ with $k$ components. Following the original construction by R.H. Fox in [8], $\Delta_{L}$ is obtained from the $(n-1) \times(n-1)$ minors of an Alexander matrix $A(L)$, which corresponds to a presentation of the link group $G(L)$ with $n$ generators and $n-1$ relators. More precisely, let $F_{n}$ denotes the free group on $n$ generators, and $H=G(L) /[G(L), G(L)]$ the abelianization of $G(L)$. The $n-1$ rows of $A(L)$ contain the $n$ formal partial derivatives of the relators of $G(L)$, first "freely" computed in $\mathbf{Z}\left[F_{n}\right]$ and then canonically mapped to $\mathbf{Z}[H] \cong \mathbf{Z}\left[t_{1}^{ \pm 1}, \ldots, t_{k}^{ \pm 1}\right]([7,8])$. In this construction we did some implicit assumptions. First we assumed a fixed ordering on the components of $L,\left(C_{1}, \ldots, C_{k}\right)$, and a fixed orientation $C_{i}^{*}$ for each of them. Further, the oriented loops $x_{1}, \ldots, x_{n}$ which generate $G(L)$ are chosen such that link $\left(x_{p}, C_{q}^{*}\right) \in\{0,+1\}$. It is known that for any $q=1, \ldots, k$ all generators $x_{p}$ with link $\left(x_{p}, C_{q}^{*}\right)=+1$ have the same image under the canonical projection $G(L) \rightarrow H$, which uniquely determines the $q$-th generator $t_{q}$ of the free abelian group $H=\left\langle t_{1}, \ldots, t_{k}\right\rangle$. So, the canonical homomorphism $a$ 
from $\mathbf{Z}\left[F_{n}\right]$ to $\mathbf{Z}[H]$ as considered in the construction of $A(L)$ is defined by the assignment $a\left(x_{p}\right)=t_{q}\left(\operatorname{link}\left(x_{p}, C_{q}^{*}\right)=1\right)$.

In the introduction we argued how to obtain $A(L)$ by dropping any row from the matrix $A(\beta)-I_{n}$, if we regard $L$ as a closed braid $L=\bar{\beta}, \beta \in B_{n}$. Such a strategy corresponds to a presentation of $G(L)$ with $n$ generators, subject to Equation (2). However, since the right hand sides of the relations in (2) might be words of considerable length, their free partial derivatives might be tedious to compute. It would be more convenient to have a direct computation scheme in terms of $\mathbf{Z}\left[t_{1}^{ \pm 1}, \ldots, t_{k}^{ \pm 1}\right]$, in order to pass by the free differential calculus. In case of knots $(k=1)$, it is well known that such a scheme is provided by the Burau representation $\tau_{B}$ of $B_{n}$ over $\mathbf{Z}\left[t, t^{-1}\right]$. Indeed, an Alexander matrix for a knot $K=\bar{\beta}$ is obtained by deleting the last (or any) row of the $n \times n$ matrix $\tau_{B}(\beta)-I_{n}$. Notice that $\tau_{B}(\beta)$ is most easily found by multiplying the basic Burau matrices $\tau_{B}\left(\sigma_{i}\right)$ in order of appearance of the braid generators $\sigma_{i}$ in a word for $\beta$. Recall that

$$
\tau_{B}\left(\sigma_{i}\right)=I_{n}-t E_{i i}+t E_{i(i+1)}+E_{(i+1) i}-E_{(i+1)(i+1)}, 1 \leq i \leq n-1,
$$

where $E_{i j}$ are the elementary matrices. A similar phenomenon can be observed for pure links, $L=\bar{\beta}$ where $\beta \in P_{n}$ is a pure braid. In this case, $k=n$ and $A(\beta)$ is exactly $\tau_{G}(\beta)$, where $\tau_{G}$ denotes the Gassner representation of $P_{n}$. But then again, in order to avoid free differential calculus in the computation of $\tau_{G}(\beta)$, one should re-express $\beta$ as a word in the pure braid generators $A_{i j}$ :

$$
A_{i j}=\sigma_{j-1} \sigma_{j-2} \cdots \sigma_{i+1} \sigma_{i}^{2} \sigma_{i+1}^{-1} \cdots \sigma_{j-2}^{-1} \sigma_{j-1}^{-1}, \quad(1 \leq i<j \leq n),
$$

after which $\tau_{G}(\beta)$ is found by multiplying the basic Gassner matrices $\tau_{G}\left(A_{i j}\right)$ in the appropriate order.

In a previous paper, [12], we offered a new method for computing $A(\beta)$ (and hence $A(L)$ ), avoiding free differential calculus, using only multiplication of matrices over $\mathbf{Z}\left[t_{1}^{ \pm 1}, \ldots, t_{k}^{ \pm 1}\right]$. In particular, this method provides a new and fast way to obtain the Gassner matrix of a pure braid without passing to pure braid generators first. To this end, we introduced multi-variable Burau matrices, the definition and main properties of which will be recalled in this section.

Let $L=C_{1}^{*} \cup \cdots \cup C_{k}^{*}$ be a link with ordered, oriented components, represented as a closed braid $L=\bar{\beta}, \beta \in B_{n}$. First, for each ordered pair $(i, j), 1 \leq i \neq j \leq n$, we consider two symbols, $s_{i j}$ and $s_{i j}^{-1}$. Each (geometric) braid $\beta \in B_{n}$ can be transformed to a word in these symbols by labeling the braid strands from left to right from 1 to $n$, where $s_{i j}$ (resp., $s_{i j}^{-1}$ ) encodes the positive (resp., negative) crossing " $i$ goes over $j$ ". In this procedure 
all crossings are processed in the order they occur in a geometric picture of $\beta$. The resulting word is called a labeled word for $\beta$, denoted by $\Psi(\beta)$. Next, we introduce the basic multi-variable Burau matrices, with entries in $\mathbf{Z}\left[t_{1}^{ \pm 1}, \ldots, t_{n}^{ \pm 1}\right]:$

$$
\begin{aligned}
\mu\left(s_{i j}\right) & =I_{n}+\left(t_{j}-1\right) E_{i i}+\left(1-t_{i}\right) E_{i j} \\
\mu\left(s_{i j}^{-1}\right)=\mu\left(s_{i j}\right)^{-1} & =I_{n}+\left(t_{j}^{-1}-1\right) E_{i i}+t_{j}^{-1}\left(t_{i}-1\right) E_{i j} .
\end{aligned}
$$

If these matrices are multiplied in the order as dictated by the word $\Psi(\beta)$, we obtain the multi-variable Burau matrix $\mu(\beta)$ for the braid $\beta$. In [12] the matrix $\mu(\beta)$ is proven to be well-defined for $\beta \in B_{n}$, that is, it is independent from the geometric representation of $\beta^{1}$.

Example. If $\beta=\sigma_{1} \sigma_{2} \sigma_{3}^{-1} \in B_{4}$, we can take $\Psi(\beta)=s_{12} s_{13} s_{41}^{-1}$.

$$
\begin{aligned}
& \mu(\beta)=\left(\begin{array}{cccc}
t_{2} & 1-t_{1} & 0 & 0 \\
0 & 1 & 0 & 0 \\
0 & 0 & 1 & 0 \\
0 & 0 & 0 & 1
\end{array}\right)\left(\begin{array}{ccccc}
t_{3} & 0 & 1-t_{1} & 0 \\
0 & 1 & 0 & 0 \\
0 & 0 & 1 & 0 \\
0 & 0 & 0 & 1
\end{array}\right) \times \\
& \left(\begin{array}{ccccc}
1 & 0 & 0 & 0 \\
0 & 1 & 0 & 0 \\
0 & 0 & 1 & 0 \\
-t_{1}^{-1}+t_{4} t_{1}^{-1} & 0 & 0 & t_{1}^{-1}
\end{array}\right) \\
& =\left(\begin{array}{cccc}
t_{2} t_{3} & 1-t_{1} & t_{2}-t_{1} t_{2} & 0 \\
0 & 1 & 0 & 0 \\
0 & 0 & 1 & 0 \\
-t_{1}^{-1}+t_{4} t_{1}^{-1} & 0 & 0 & t_{1}^{-1}
\end{array}\right) .
\end{aligned}
$$

If we specialize $t_{1}=t_{2}=\ldots=t_{n}=t$ in $\mu(\beta)$ we get the Burau matrix $\tau_{B}(\beta)$ of $\beta$ up to a permutation of the columns, motivating the name "multivariable Burau matrix". Furthermore, if $\beta$ is a pure braid then $\mu(\beta)$ is exactly the Gassner matrix $\tau_{G}(\beta)$. But even if $\beta \notin P_{n}, \mu(\beta)$ immediately leads to an Alexander matrix for $L$. In order to formulate all this in a proper way, we need the canonical homomorphism from the braid group $B_{n}$ to the symmetric group $S_{n}$, determined by

$$
p: B_{n} \rightarrow S_{n}: \sigma_{i} \rightarrow(i, i+1) \quad(1 \leq i \leq n-1) .
$$

Observe that the number of disjoint cycles of $p(\beta)$ equals $k$, the number of components of $L=\bar{\beta}$. For each strand label $1 \leq i \leq n$ we put $c(i)$ equal

\footnotetext{
${ }^{1}$ As a matter of fact, we introduced relations on the $s_{i j}$ and $s_{i j}^{-1}$, yielding a presentation of a group called $L B_{n}$, the labeled braid group. This makes $\Psi$ a well-defined mapping from $B_{n}$ to $L B_{n}$, and moreover, $\mu$ is a linear group representation for $L B_{n}$ over $\mathbf{Z}\left[t_{1}^{ \pm 1}, \ldots, t_{n}^{ \pm 1}\right]$.
} 
to the label of the component of $L$ it belongs to in the braid closure. The substitution

$$
t_{i} \rightarrow t_{c(i)}
$$

extends to a ring homomorphism, called the component specializer:

$$
c: \mathbf{Z}\left[t_{1}^{ \pm 1}, \ldots, t_{n}^{ \pm 1}\right] \rightarrow \mathbf{Z}\left[t_{1}^{ \pm 1}, \ldots, t_{k}^{ \pm 1}\right] .
$$

Its entry-wise action on the matrix $\mu(\beta)$ will be denoted by $\mu(\beta)^{c}$. Notice that for pure braids $\beta c$ is just a permutation of the variables $t_{i}(k=n)$. In the next theorem, $\pi_{\rho}$ denotes the standard $n \times n$ matrix representing the permutation $\rho \in S_{n}$.

Theorem 1. Let $L=C_{1}^{*} \cup \cdots \cup C_{k}^{*}$ be a link with $k$ ordered, oriented components, represented as closed braid, $L=\bar{\beta}$. Then

$$
A(\beta)=\mu(\beta)^{c} \cdot \pi_{p(\beta)} .
$$

In particular, if $\mu_{t}(\beta)$ is the image of $\mu(\beta)$ under the specialization $t_{1}=$ $\ldots=t_{n}=t$,

$$
\tau_{B}(\beta)=\mu_{t}(\beta) \cdot \pi_{p(\beta)},
$$

and if $\beta$ is a pure braid,

$$
\tau_{G}(\beta)=\mu(\beta)
$$

Proof. The connection between $\mu(\beta)$ and the Burau and Gassner representations has been elaborated in [12]. The general statement, which relates $A(\beta)$ to $\mu(\beta)^{c}$ is implicitly present in the same article. It follows from the construction of $\mu(\beta)$ as the Magnus representation corresponding to a representation $\varphi$ of the labeled braid group $L B_{n}$ into $\operatorname{Aut}\left(F_{n}\right)$, having the property

$$
\pi_{p(\beta)} \circ \varphi(\Psi(\beta))=\xi(\beta),
$$

where $\xi$ is Artin's representation, and where $\pi_{p(\beta)}$ is now regarded as a free automorphism.

\section{Isotopy of Line Configurations.}

In this section we exhibit the motivating problem behind this paper, namely the classification of line configurations up to (rigid) isotopy. A configuration of $n$ lines $\mathbf{R P}^{3}$ is called non-singular if no pair intersects in projective space. Two such configurations, $\mathcal{L}=\left\{L_{1}, \ldots, L_{n}\right\}$ and $\mathcal{M}=\left\{M_{1}, \ldots M_{n}\right\}$, 
are called rigidly isotopic if there exists a homotopy $\left(h_{t}\right)_{t \in[0,1]}$ of the ambient space such that $h_{0}=\mathrm{id}, h_{1}(\mathcal{L})=\mathcal{M}$ and $h_{t}(\mathcal{L})$ is a non-singular line configuration for all $t$. Furthermore, we can consider (rigid) isotopies of ordered configurations, where we require that $h_{1}\left(L_{i}\right)=M_{i}$ for $1 \leq i \leq n$. The isotopy classification of (unordered) line configurations for $n \leq 5$ has been accomplished by O.Ya. Viro in [16], for $n=6$ by V. Mazurovskii in [9], and recently for $n=7$ by A. Borobia and V. Mazurovskii in [4]. For a treatment of line isotopy from the view point of projective geometry we refer to [5]. The connection between non-singular line configurations and links in $\mathbf{R}^{3}$ is straightforward. Indeed, lines in $\mathbf{R P}^{3}$ can be pulled back to circles in $S^{3}$, which in their turn can be stereographically projected from the north pole of $S^{3}$ to circles in $\mathbf{R}^{3}$. By this way, a non-singular line configuration $\mathcal{L}$ is mapped to a configuration $\gamma(\mathcal{L})$ of mutually linked circles, called the Klein link associated with $\mathcal{L}$. See [5] for more details. It is a natural relaxation to consider flexible isotopies of the associated (topological) link $\gamma(\mathcal{L})$ rather than rigid isotopies of the (geometric) configuration $\mathcal{L}$. We know that for $n \leq 7$ the flexible isotopy classification of line configurations coincides with the rigid isotopy classification.

If we choose the plane at infinity such that it does not contain any of the lines of $\mathcal{L}$ then we can regard $\mathcal{L}$ as a configuration of $n$ mutually skew lines in $\mathbf{R}^{3}$. The planar layout of this affine part is a projection of the lines upon a plane in $\mathbf{R}^{3}$ such that no line becomes a point, no pair becomes parallel and no triple becomes concurrent. Furthermore, we visualize over and under crossings at the intersections in a planar layout in the same fashion as in knot and braid diagrams (Fig. 1).

Let us now describe the procedure to obtain a diagram of the associated Klein link $\gamma(\mathcal{L})$ from a given planar layout of $\mathcal{L}$. Imagine a circle $U$ drawn in the plane which contains all crossings of the planar layout of $\mathcal{L}$. Choose an arbitrary point $p$ on the circle $U$. This determines an order on the lines, namely, the order in which the lines are encountered during a counterclockwise trip on $U$ starting at $p$. At the same time, this "pointed circle" $(U, p)$ yields an orientation of the lines, by directing the lines from their "first" intersection with $U$ toward the "second" intersection. See Fig. 1 for an ordering and orientations in a planar layout, coming from a certain choice of $p$ on $U$. Notice that if one is only given the order of the lines, without knowledge of the point $p$ on $U$, ambiguity appears in the sense that the resulting orientations of the lines are only determined now up to total reversal. We will use the term semi-orientation in this context. Now it should be obvious how to turn this picture into a (geometric) braid, as in Fig. 1. Let $\alpha=\alpha(\mathcal{L})$ denote the braid thus obtained (implicitly depending on the choice for $p$ !). If a braid can be obtained in this way, it is called a line braid. The following 

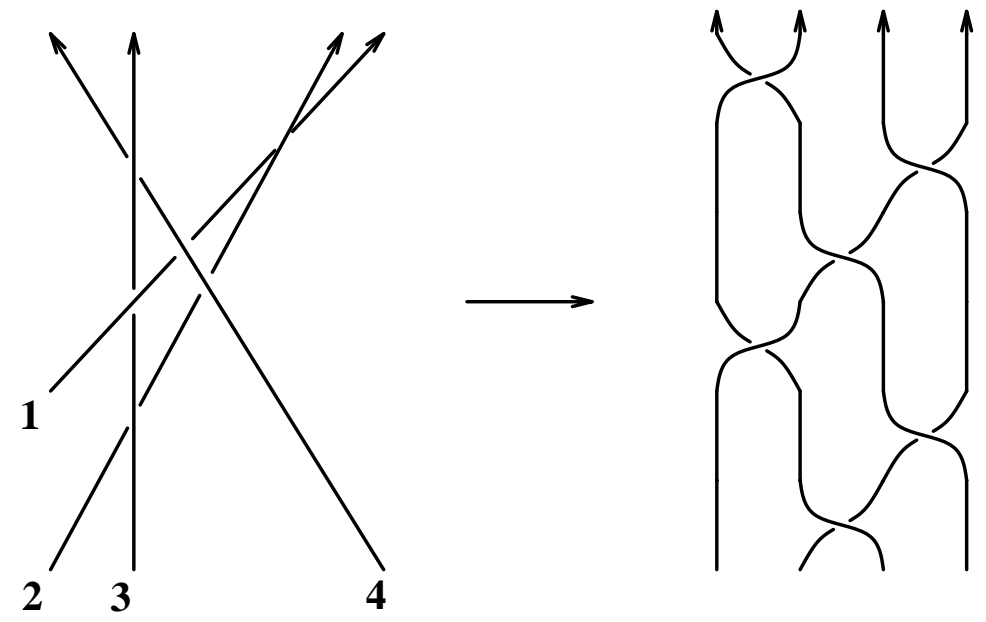

Figure 1: The planar layout of four skew lines in 3-space. The chosen order of the lines determines the shown braid at the right (braid diagrams are oriented from bottom to top in this article).

operation will be used to turn line braids into pure braids:

$$
\text { ^ } B_{n} \rightarrow B_{n}: \sigma_{i} \rightarrow \sigma_{n-i} .
$$

By checking the defining relations of the Braid group, the reader can convince himself that ${ }^{\wedge}$ can be extended to an automorphism of $B_{n}$. To support the intuition, the effect of ${ }^{\wedge}$ on a geometric braid $\beta$ represented in the cylinder $D^{2} \times I$ is a rotation of $\beta$ of $180^{\circ}$ around the cylinder axis. Therefore, ${ }^{\wedge}$ is called the flip operator. Now we can introduce the double of a line braid by taking $\alpha \hat{\alpha}$, where $\alpha \in B_{n}$. It is straightforward that the double of a line braid is a pure braid, and so, its closure can be regarded as an ordered link, the components of which have inherited their labels from the braid strands. In [5] it is proven that if $\alpha=\alpha(\mathcal{L})$ then the closure of $\alpha \hat{\alpha}$ represents the link type of $\gamma(\mathcal{L})^{2}$, yielding a procedure to draw a diagram for $\gamma(\mathcal{L})$ when a planar layout for $\mathcal{L}$ is given (Fig. 2). In fact, this diagram transformation is a special case of a general procedure to go from projective diagrams of links in $\mathbf{R P}^{3}$ to the affine diagram of the twofold covering in $S^{3}$, as described by Yu.V. Drobotukhina in [6].

Notice that an orientation $A^{*}$ for a line $A$ of $\mathcal{L}$ leads to an orientation $\gamma(A)^{*}$ of the corresponding component of the link $\gamma(\mathcal{L})$. If $\left\{A^{*}, B^{*}\right\}$ is a

\footnotetext{
${ }^{2}$ More precisely, the mirror image of the closure of $\alpha \hat{\alpha}$ represents $\gamma(\mathcal{L})$, if we use the same projection for the link diagram as for the planar layout [5].
} 


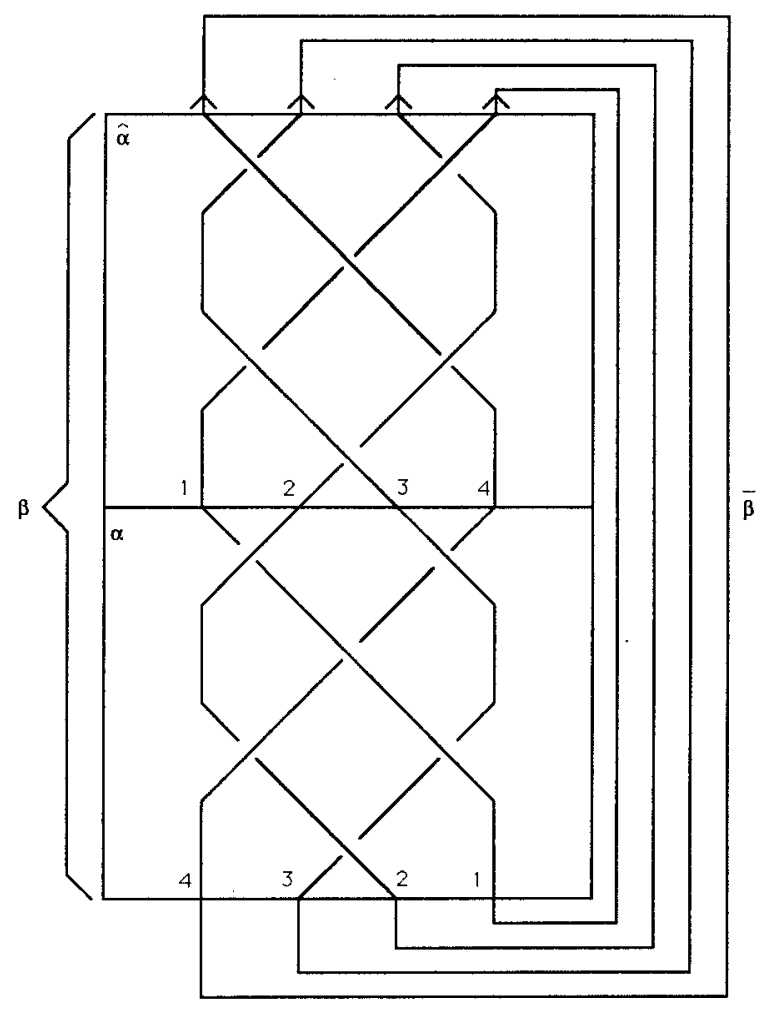

Figure 2: After doubling the line braid of a configuration $\mathcal{L}$, we obtain a diagram for the associated link $\gamma(\mathcal{L})$.

pair of oriented skew lines in 3-space, by their linking number we mean link $\left(\gamma(A)^{*}, \gamma(B)^{*}\right)$.

We define the Alexander polynomial of an ordered, non-singular configuration $\mathcal{L}$ of $n$ oriented lines in $\mathbf{R P}^{3}$ to be the multi-variable Alexander polynomial of the link $\gamma(\mathcal{L})$, where the latter inherits its labels and orientations from $\mathcal{L}$. So, it is a Laurent polynomial in $n$ variables with integer coefficients, only defined up to multiplication by units in $\mathbf{Z}\left[t_{1}^{ \pm 1}, \ldots, t_{n}^{ \pm 1}\right]$ (see $[8]$ ), and it will be denoted by $\Delta_{\mathcal{L}}\left(t_{1}, \ldots, t_{n}\right)$. In the Appendix we give a list of all rigid isotopy types of line configurations for $n \leq 6$, up to taking mirror images. This list follows from Viro's and Mazurovskii's classification efforts. For each configuration in the Appendix, we computed the Alexander polynomial for one particular choice of orientations and labels. For other orderings one should perform the associated permutation on the variables of 
$\Delta_{\mathcal{L}}\left(t_{1}, \ldots, t_{n}\right)$, while a change of orientation corresponds to substituting the involved variables $t_{i}$ by $t_{i}^{-1}$ (cf. [14]). Furthermore, if $\mathcal{L}^{m}$ denotes the mirror of $\mathcal{L}$, obtained by changing in the planar layout of $\mathcal{L}$ all under crossings into over crossings (and vice versa) and keeping the same labels and orientations, it is well known that

$$
\Delta_{\mathcal{L}} \doteq \Delta_{\mathcal{L}^{m}} .^{3}
$$

We obtained the Alexander polynomials of the Appendix by first choosing a line braid $\alpha=\alpha(\mathcal{L})$ for $\mathcal{L}$, which determines some particular ordering and orientation for its lines, and by using the multi-variable Burau matrices of Section 2 to compute the Gassner matrix for $\alpha \hat{\alpha}$. Finally, from the latter we get an Alexander matrix. We preferred to represent the Alexander polynomial with positive powers for its variables, thus being an ordinary polynomial, requiring moreover minimal degree. This determines $\Delta_{\mathcal{L}}$ up to sign. Observe that some configurations appear to have a very simple Alexander polynomial, while those of other configurations tend to be of considerable length. The geometric reason for this phenomenon will be explained in Section 6 .

The $\Delta_{\mathcal{L}}$ of two ordered configurations can be compared by regarding the canonical (semi-)orientations for which the last line has all positive linking numbers w.r.t. the other lines. It should be remarked that the multi-variable Alexander polynomial does not classify ordered configurations, even not up to mirror images. We found the first failure of $\Delta_{\mathcal{L}}$ for the fifth 6-linesconfiguration in the Appendix (configuration [6-12-8-2] in [5]), where the transposition (2 3 ) on the ordering yields a different isotopy type (distinguished by chirality) but leaves $\Delta_{\mathcal{L}}$ invariant.

On the other hand, in [12] an example is given of a pair of ordered configurations of seven lines, both of the same chirality class and having the same Kauffman polynomial, but distinguished by $\Delta_{\mathcal{L}}$.

\section{The path model.}

In this section we give a topological construction for the entries of $\mu(\beta)$, the multi-variable Burau matrix of the braid $\beta$. In particular, it provides a topological model for the Gassner representation. In the next section we see the use of this model in understanding the appearance of some factors in $\Delta_{\mathcal{L}}$.

Let $\beta \in B_{n}$ be a braid, geometrically represented by a diagram in the plane, as in Figure 3. Let $h_{1}, \ldots, h_{n}$ be the (oriented) braid strands in this diagram. A (proper) path of $\beta$ is a continuous path on $h_{1} \cup \ldots \cup h_{n}$ starting at the initial point of some $h_{i}$, never violating the braid strand orientations

\footnotetext{
${ }^{3}$ Everywhere in this article, "” means "equal up to a multiplicative unit".
} 
(monotone), only allowed to make a "turn" (change underlying strands) from an over crossing strand to an under crossing strand, arriving at the end point of some $h_{j}$. In this case, we say that the path "goes from $i$ to $j$ ". See Figure 3 for an illustration of a path that goes from 4 to 4 . Notice that each braid strand $h_{i}$ must be regarded as a proper path, without turning points, and will be sometimes referred to as a straight path.

A path $\pi$ on a braid diagram can be combinatorially encoded as follows. Let $w=s_{i j}^{ \pm 1} \ldots$ be a labeled word associated with (the diagram representing) $\beta \in B_{n}$. Considering $w$ as an ordered sequence, the turning points of a path $\pi$ of $\beta$ occur as an ordered subsequence $T(\pi)$ of $w$ with the property that if $s_{p q}^{ \pm 1}$ and $s_{r s}^{ \pm 1}$ are two successive symbols of $T(\pi)$ then $q=r$. If $s_{a b}^{ \pm 1}$ is the first symbol of $T(\pi)$ and $s_{c d}^{ \pm 1}$ the last one, then $\pi$ goes from $a$ to $d$. There is a one-to-one correspondence between proper non-straight paths on a braid diagram and non-empty ordered subsets $T$ of the associated labeled word $w$ that have the above properties; this correspondence is given by $\pi \rightarrow T(\pi)$. Notice that for straight paths $T\left(h_{i}\right)=\emptyset$. The set of all paths of $w$ going from $a$ to $b$ is denoted by $P_{a b}$ (or $P_{a b}(w)$ when there is danger for confusion).

The complement of a sequence of turning points $T(\pi)$ in $w, w \backslash T(\pi)$, is an ordered subsequence of $w$, which is partitioned by $T(\pi)$ in a natural fashion:

$$
w \backslash T(\pi)=\left(I_{1}, \ldots, I_{k}\right),
$$

where the symbols of each $I_{i}$ occur successively in $w$, but where the last symbol of $I_{i}$ is separated from the first symbol of $I_{i+1}$ in $w$ by at least one turning point of $\pi$. These partition classes are called intervals of $\pi$. If $I$ is such an interval of a non-straight path $\pi$, and if $s_{p q}^{ \pm 1}$ and $s_{q r}^{ \pm 1}$ are two adjacent turning points of $\pi$ such that $\left(s_{p q}^{ \pm 1}, I, s_{q r}^{ \pm 1}\right)$ occurs successively in $w$, then $s_{p q}^{ \pm 1}$ (resp., $s_{q r}^{ \pm 1}$ ) is called the q-entry (resp., q-exit) of $I$. Notice that the initial (resp., final) interval of $\pi, I_{1}$ (resp., $I_{k}$ ), may cease to have an entry (resp., exit), but that in any case they have at least one of both. This enables us to say that $I$ is a $q$-interval of $\pi$ if it has a $q$-entry or a $q$-exit. Notice that a path $\pi$ may have different $q$-intervals for the same $q$. A straight path $h_{i}$ has only one interval, namely the whole $w$, which is called the $i$-interval of $h_{i}$ by convention. If a symbol $s_{i q}^{ \pm 1}$ (resp., $s_{q i}^{ \pm 1}$ ) of $w$ belongs to a $q$-interval of some path $\pi$ (so it is surely no turning point of $\pi$ ) then we say that it is an under crossing (resp., over crossing) of $\pi$. In particular, every $s_{i j}^{ \pm 1}$ (resp., $s_{j i}^{ \pm 1}$ ) of $w$ is an over crossing (resp., under crossing) of the straight path $h_{i}$.

To illustrate these definitions, let us consider one of those typical braids we encounter in the study of line configurations (Section 3), namely, the "double of a line braid" (Fig. 3). The given diagram representation corresponds to the labeled word

$$
s_{12} s_{45} s_{35} s_{15} s_{52}^{-1} s_{34} s_{14} s_{31}^{-1} s_{42}^{-1} s_{32}^{-1} s_{21} s_{54} s_{53} s_{51} s_{25}^{-1} s_{43} s_{41} s_{13}^{-1} s_{24}^{-1} s_{23}^{-1} .
$$




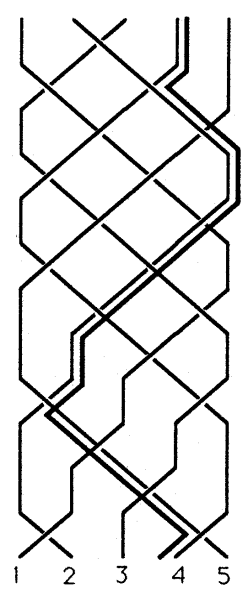

Figure 3: The topological meaning of a "path".

In Figure 3 we selected the path $\pi$ in $P_{44}$ whose turning points are given by

$$
T(\pi)=\left(s_{45}, s_{52}^{-1}, s_{24}^{-1}\right),
$$

its under crossings by

$$
\left(s_{35}, s_{15}, s_{42}^{-1}, s_{32}^{-1}\right)
$$

and its over crossings by

$$
\left(s_{21}, s_{25}^{-1}\right) .
$$

Observe that $\pi$ has two 4-intervals (initial and final), a 5-interval and a 2 -interval. For example, the 2-interval has entry $s_{52}^{-1}$ and exit $s_{24}^{-1}$.

Let us now take the turning points and over/under crossings of a path $\pi$ all together in one ordered subsequence of $w$, denoted by $V(\pi)$, called the vertices of $\pi$. To distinguish the turning points in $V(\pi)$ we will denote them by symbols $u_{i j}^{ \pm 1}$ rather than $s_{i j}^{ \pm 1}$. For example, the vertices of the path $\pi \in P_{44}$ in Figure 3 are given by

$$
V(\pi)=\left(u_{45}, s_{35}, s_{15}, u_{52}^{-1}, s_{42}^{-1}, s_{32}^{-1}, s_{21}, s_{25}^{-1}, u_{24}^{-1}\right) .
$$

Each subsequence of $w$, with some of $s_{i j}^{ \pm 1}$ replaced by $u_{i j}^{-1}$, which occurs as $V(\pi)$ for some path $\pi$ is called a combinatorial path. We will often confuse between a path $\pi$ and its combinatorial description $V(\pi)$.

Next, we define for each vertex $v$ of $\pi$ a weight $W(v, \pi)$ (or $W(v)$ if there is no confusion about the considered path), an element of the ring 
$\mathbf{Z}\left[t_{1}^{ \pm 1}, \ldots, t_{n}^{ \pm 1}\right]$, as follows:

$$
\begin{aligned}
v \text { is an under crossing of } \pi: & W(v)=1 \\
v \text { is an over crossing of } \pi: v & =s_{i j}: W(v)=t_{j} \\
v & =s_{i j}^{-1}: W(v)=t_{j}^{-1} \\
v \text { is a turning point of } \pi: v & =u_{i j}: W(v)=1-t_{i} \\
v & =u_{i j}^{-1}: W(v)=t_{j}^{-1}\left(t_{i}-1\right) .
\end{aligned}
$$

Now we can define the weight of a path

$$
[\pi]=\prod_{v \in V(\pi)} W(v)
$$

and further, for each word $w$ we define the polynomials

$$
[i j]_{w}=[i j]=\sum_{\pi \in P_{i j}}[\pi]
$$

If $V(\pi)$ is empty, which may happen when $\pi=h_{i}$, then we define $[\pi]=1$. If $P_{i j}=\emptyset$ then we define $[i j]=0$. In [12] we proved the following theorem, which will be referred to as the path model for multi-variable Burau matrices.

Theorem 2. Let $\beta \in B_{n}$ be a braid with labeled word $w$. The entries of its multi-variable Burau matrix are given by

$$
\mu(\beta)_{i j}=[i j]_{w}
$$

\section{Grasp extensions.}

For a braid $\beta \in B_{n-1}$ we can consider its grasp extension, which is a braid $\beta^{g} \in B_{n}$ that is defined by

$$
\beta^{g}=\beta \sigma_{n-1} \sigma_{n-2} \cdots \sigma_{1}^{2} \sigma_{2} \cdots \sigma_{n-1} .
$$

We refer to Figure 4 for a diagram representation of a grasp extension.

In [12] it has been observed that each line configuration is rigidly isotopic to a configuration $\mathcal{L}$ where the $n$-th line crosses under the remaining $n-1$ lines (w.r.t. the given projection). If now we choose orientations, $\mathcal{L}^{*}=$ $\left\{L_{1}^{*}, \ldots, L_{n}^{*}\right\}$, with the property that

$$
\operatorname{link}\left(L_{i}^{*}, L_{n}^{*}\right)=+1, \quad 1 \leq i \leq n-1
$$




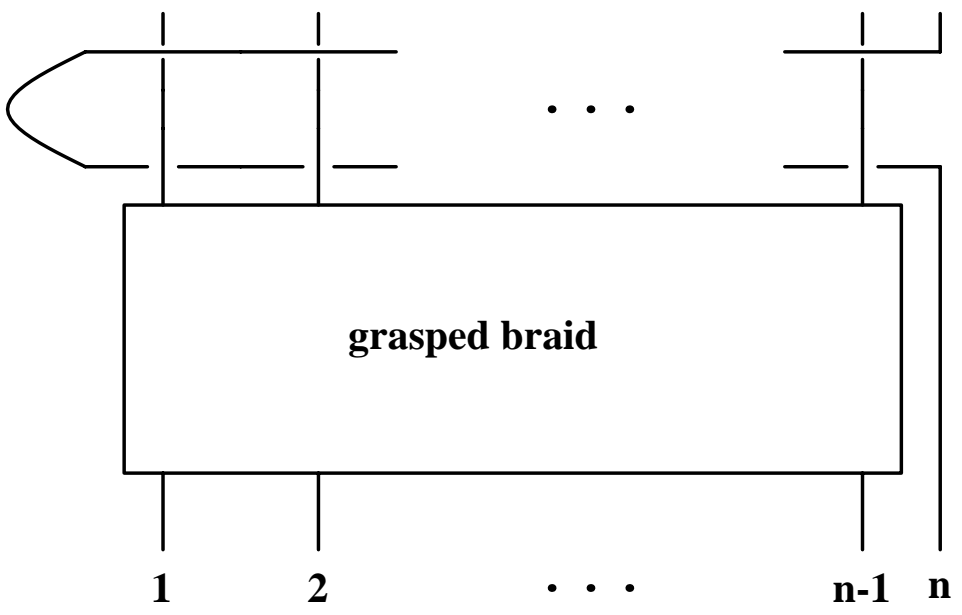

Figure 4: The grasp extension of a braid. The $n$-th string $h_{n}$ of $\beta^{g}$ grasps the strings of $\beta$.

this determines line braids $\alpha_{n}=\alpha\left(\mathcal{L}^{*}\right)$ and $\alpha_{n-1}=\alpha\left(\mathcal{L}^{*} \backslash\left\{L_{n}^{*}\right\}\right)$, such that the double braid $\beta_{n}=\alpha_{n} \widehat{\alpha_{n}}$ is the grasp extension of the double braid $\beta_{n-1}=\alpha_{n-1} \widehat{\alpha_{n-1}}$ :

$$
\beta_{n}=\beta_{n-1}^{g} .
$$

Recall from Section 3 that the associated Klein link $\gamma\left(\mathcal{L}^{*}\right)$ can be obtained as the closure of $\beta_{n}{ }^{4}$, and that the Alexander polynomial $\Delta_{\mathcal{L}^{*}}$ can be computed by means of the multi-variable Burau matrix $\mu\left(\beta_{n}\right)$.

In this section we try to relate $\mu(\beta)$ to $\mu\left(\beta^{g}\right)$, a goal which is motivated by the previous remarks.

Theorem 3. Let $\beta \in B_{n-1}$. If $[i j]$ and $[i j]^{g}$ denote the $(i, j)$-entries of $\mu(\beta)$ and $\mu\left(\beta^{g}\right)$, respectively, and if we use the shorthands

$$
S_{i}=\sum_{k=1}^{n-1}\left(1-t_{k}\right)[i k]
$$

\footnotetext{
${ }^{4}$ Following Morton's terminology, $\gamma\left(\mathcal{L}^{*}\right)$ may be equally well regarded as the complete closure of $\beta_{n-1}([\mathbf{1 1}])$.
} 
then $(1 \leq i, j \leq n-1)$ :

$$
\begin{aligned}
{[i j]^{g} } & =t_{n}[i j]+S_{i} t_{1} \cdots t_{j-1}\left(1-t_{n}\right) \\
{[i n]^{g} } & =S_{i} t_{1} \cdots t_{n-1} \\
{[n j]^{g} } & =t_{1} \cdots t_{j-1}\left(1-t_{n}\right) \\
{[n n]^{g} } & =t_{1} \cdots t_{n-1} .
\end{aligned}
$$

Proof. Choose labeled words $w$ and $w^{g}$ for $\beta$ and $\beta^{g}$, respectively, compatible with the diagram in Figure 4 or Equation 14:

$$
w^{g}=w s_{(n-1) n} \cdots s_{1 n} s_{n 1} \cdots s_{n(n-1)} .
$$

Further, let $P_{i j}$ and $P_{i j}^{g}$ denote the corresponding path sets.

Because $P_{n n}^{g}=\left\{h_{n}\right\}$, we get

$$
[n n]^{g}=\left[h_{n}\right]=t_{1} \cdots t_{n-1} .
$$

Further, for each $1 \leq j \leq n-1, P_{n j}^{g}=\{\pi\}$ with $T(\pi)=\left\{s_{n j}\right\}$, whence

$$
\begin{aligned}
{[n j]^{g}=[\pi] } & =t_{1} \cdots t_{j-1}\left(1-t_{n}\right) \text { if } j>1 \\
{[n 1]^{g} } & =1-t_{n} .
\end{aligned}
$$

Next, let $1 \leq i \leq n-1$, then for each $1 \leq k<n$ and each $\pi \in P_{i k}$, we obtain a path $\pi^{g} \in P_{i j}^{g}$ by the following concatenation rule:

$$
\pi^{g}=\pi\left(s_{k n}, \ldots, s_{1 n}, s_{n 1}, \ldots, s_{n j}\right) .
$$

Notice that $T\left(\pi^{g}\right)=T(\pi) \cup\left\{s_{k n}, s_{n j}\right\}$. Conversely, each path in $P_{i j}^{g}$ is either of this type, or of the form

$$
\pi^{c}=\pi\left(s_{j n}, s_{n j}\right),
$$

the straight continuation of some $\pi \in P_{i j}$ (Figure 4). This leads to the expression

$$
[i j]^{g}=[i j] t_{n}+\sum_{k=1}^{n-1}[i k]\left(1-t_{k}\right) t_{1} \cdots t_{j-1}\left(1-t_{n}\right) .
$$

Finally, each path in $P_{i n}^{g}$ can be obtained as in Equation 15, where we now continue tracing $h_{n}$ till the end (that is, $s_{n(n-1)}$ ) instead of making a turn at $s_{n j}$. This means that $T\left(\pi^{g}\right)=T(\pi) \cup\left\{s_{k n}\right\}$, and so,

$$
[i n]^{g}=\sum_{k=1}^{n-1}[i k]\left(1-t_{k}\right) t_{1} \cdots t_{n-1} .
$$


As a corollary we can state (recall that the component specializer $c$ is defined by $c\left(t_{i}\right)=t_{q}$ if the $i$-th string of $\beta$ contributes to the $q$-th component of the closure $\bar{\beta})$ :

Theorem 4. If $\beta^{g} \in B_{n}$ is the grasp extension of $\beta \in B_{n-1}$, and if its closure represents the (oriented) link $L$, an Alexander matrix $A(L)$ for $L$ can be obtained by deleting an arbitrary row of the matrix

$$
\left(\begin{array}{c|c}
t_{n} \mu(\beta)-I_{n-1} & S_{1} \\
& S_{n-1} \\
\hline[n 1]^{g} \cdots[n(n-1)]^{g} & t_{1} \cdots t_{n-1}-1
\end{array}\right),
$$

and applying the component specializer c entry-wise.

Proof. As explained in Section 2, an Alexander matrix for $L$ can be computed by deleting any row from $\mu\left(\beta^{g}\right)^{c}-I_{n}$, or from a matrix obtained from this by elementary row operations. Since applying $c$ commutes with subtracting by $I_{n}$, row deletion, and elementary row operations, we can first consider $M=\mu\left(\beta^{g}\right)-I_{n}$. If $R_{i}$ denotes the $i$-th row of $M$, we can do the following row operations on $M$ :

$$
R_{i} \rightarrow R_{i}-S_{i} \cdot R_{n}, \quad 1 \leq i \leq n-1
$$

As before, we have put

$$
S_{i}=\sum_{k=1}^{n-1}\left(1-t_{k}\right)[i k] .
$$

From Theorem 3 it follows that the resulting matrix is given by (16).

\section{Remarks.}

- If we delete the last row in (16), and if we compute the Alexander polynomial $\Delta_{L}$ by means of the minor complementary to the last column, then we rediscover a classical theorem of Morton on "complete closures" ([10], and also [12]):

$$
\Delta_{L} \cdot\left(t_{n}-1\right) \doteq \operatorname{det}\left(\mu(\beta)^{c}-t_{k}^{-1} I_{n-1}\right),
$$

where we assumed that $c\left(t_{n}\right)=t_{k}$, and used that

$$
c\left(\operatorname{det}\left(\mu(\beta)-t_{n}^{-1} I_{n-1}\right)\right)= \pm \operatorname{det}\left(\mu(\beta)^{c}-t_{k}^{-1} I_{n-1}\right) .
$$


- On the other hand, if we delete another row in (16) (not the last row), and if we now compute $\Delta_{L}$ by taking the minor complementary to any column (not the last one), then we find a classical theorem of Torres, stated here in the case of complete closures only, [14] (assume $\left.c\left(t_{n}\right)=t_{k}\right)$ :

$$
\Delta_{L}\left(t_{1}, \ldots, t_{k-1}, 1\right) \doteq\left(c\left(t_{1} \cdots t_{n-1}\right)-1\right) \cdot \Delta_{\bar{\beta}}\left(t_{1}, \ldots, t_{k-1}\right) .
$$

Indeed, substituting $t_{n}=1$ in (16) gives:

$$
\left(\begin{array}{c|c}
\mu(\beta)-I_{n-1} & * \\
& \vdots \\
\hline 0 \ldots 0 & t_{1} \cdots t_{n-1}-1
\end{array}\right) .
$$

\section{The Alexander polynomial of a configuration with adjacent lines.}

Following O.Ya. Viro $([\mathbf{1 6}, \mathbf{1 7}])$, two lines $A$ and $B$ of a non-singular line configuration $\mathcal{L}$ in $\mathbf{R P}^{3}$ are called adjacent if there exists a rigid isotopy of $\mathcal{L}$ which brings $A$ and $B$ close enough to each other such that they are separated from the other lines by a hyperboloid. Notice that once $A$ and $B$ are in the latter position they can be brought arbitrarily close to each other by a rigid isotopy of the entire configuration such that they coincide "in the limit" (see [5] for a formal treatment of limit-isotopy). Obviously, if $A$ and $B$ are adjacent in $\mathcal{L}$, the configurations $\mathcal{L} \backslash\{A\}$ and $\mathcal{L} \backslash\{B\}$ are rigidly isotopic; their isotopy class can be considered to be the limit-configuration where $A$ and $B$ coincide, denoted by $\mathcal{L} /(A=B)$.

In this section we deduce the Alexander polynomial of $\mathcal{L}$ from the Alexander polynomial of $\mathcal{L} /(A=B)$. If we consider the associated links, $\gamma(\mathcal{L})$ is obtained from $\gamma(\mathcal{L} /(A=B))$ by what topologists are used to call a cabling process. More precisely, we can orient a pair of adjacent lines such that they become homologous as oriented cycles, $\gamma(A)^{*}$ and $\gamma(B)^{*}$, in the complement of $\gamma(\mathcal{L} \backslash\{A, B\})$, whence $\gamma(B)^{*}$ is a $(1,1)$-cable or $(1,-1)$-cable of $\gamma(A)^{*}$, if link $\left(A^{*}, B^{*}\right)=1$ or -1 respectively. In fact, Theorem 5 appears to be a special case of the result of D.W. Sumners and J.M. Woods in 1977 for general $(p, q)$-cables $([\mathbf{1 3}])$. In their terminology, $\mathcal{L}$ is an iteration of type 2 of $\mathcal{L} /(A=B)$. However, their proof is certainly not straightforward, motivating V.G. Turaev to give his proof in 1986, which appeals to the concept of Reidemeister torsion ([15]). As another example of the elegance of the path model, we will analyze the effect of the "cabling-process" on the multivariable Burau matrix, thus yielding a combinatorial (third!) proof of this 
classical result. Everything will be stated for line configurations, since this is our case of interest, although a more general result should be obtained by a slight extension of our proof (e.g., in the case of $(1, q)$-cables).

Since the Alexander polynomial is an isotopy invariant, we may assume that the adjacent lines $A$ and $B$ are sufficiently close to each other such that in the planar layout of $\mathcal{L}, A$ and $B$ are consecutively met by a circle containing all crossings. This means that we can choose a corresponding labeling for $\mathcal{L}$ such that lines $A$ and $B$ occur with labels $n-1$ and $n$, respectively. Furthermore, we may assume for each $C \in \mathcal{L} \backslash\{A, B\}$ that $C$ crosses over $A$ and $C$ crosses over $B$ (in the planar layout). This is a consequence of the previously cited theorem in [12], and of the fact that $A$ and $B$ may be separated from the remaining $n-2$ lines by a hyperboloid. For the associated (semi-)orientation of $\mathcal{L}$ we see that for each $C^{*} \in \mathcal{L}^{*} \backslash$ $\left\{A^{*}, B^{*}\right\}:^{5}$

$$
\operatorname{link}\left(A^{*}, C^{*}\right)=\operatorname{link}\left(B^{*}, C^{*}\right)=+1 .
$$

We will consider the Alexander polynomial of $\mathcal{L}$ subject to the previously chosen labeling and semi-orientation, while for $\mathcal{L} /(A=B)$ we omit the $n$-th line, keeping the remaining labels and orientations. Our computations will go by way of the Gassner matrices of the double braids $\beta_{n}=\beta\left(\mathcal{L}^{*}\right)$ and $\beta_{n-1}=\beta\left((\mathcal{L} / A=B)^{*}\right)$, where the latter equals the former with the $n$-th string deleted. Observe that $\beta\left(\mathcal{L}^{*}\right)$ is only determined up to total reversal by the previous choices, but that this does not affect the Alexander polynomial. Finally, let $\beta_{n-2}$ denote the double braid associated with $\mathcal{L}^{*} \backslash\left\{A^{*}, B^{*}\right\}$, that is, $\beta_{n-1}$ with the $(n-1)$-th string deleted (Figure 5$)$.

After these conventions we now introduce some terminology in order to state the main theorem. First, let us define the iterator $F$ as the ring homomorphism from $\mathbf{Z}\left[t_{1}, t_{1}^{-1}, \ldots, t_{n-1}, t_{n-1}^{-1}\right]$ to $\mathbf{Z}\left[t_{1}, t_{1}^{-1}, \ldots, t_{n}, t_{n}^{-1}\right]$ which is determined by

$$
\begin{aligned}
F\left(t_{i}\right) & =t_{i}, \quad 1 \leq i<n-1, \\
F\left(t_{n-1}\right) & =t_{n-1} t_{n} .
\end{aligned}
$$

Next, if $1 \leq i<n-1$ is the label for $C \in \mathcal{L} \backslash\{A, B\}$, then, for the given orientation $\mathcal{L}^{*}$, we put

$$
\begin{aligned}
l_{i} & =\operatorname{link}\left(A^{*}, C^{*}\right)=\operatorname{link}\left(B^{*}, C^{*}\right), \\
l & =\operatorname{link}\left(A^{*}, B^{*}\right) .
\end{aligned}
$$

\footnotetext{
${ }^{5}$ The first equality is exactly equivalent to the fact that $\gamma(A)^{*}$ and $\gamma(B)^{*}$ are homologous as oriented cycles in the complement of $\gamma(\mathcal{L}) \backslash\{\gamma(A), \gamma(B)\}$.
} 


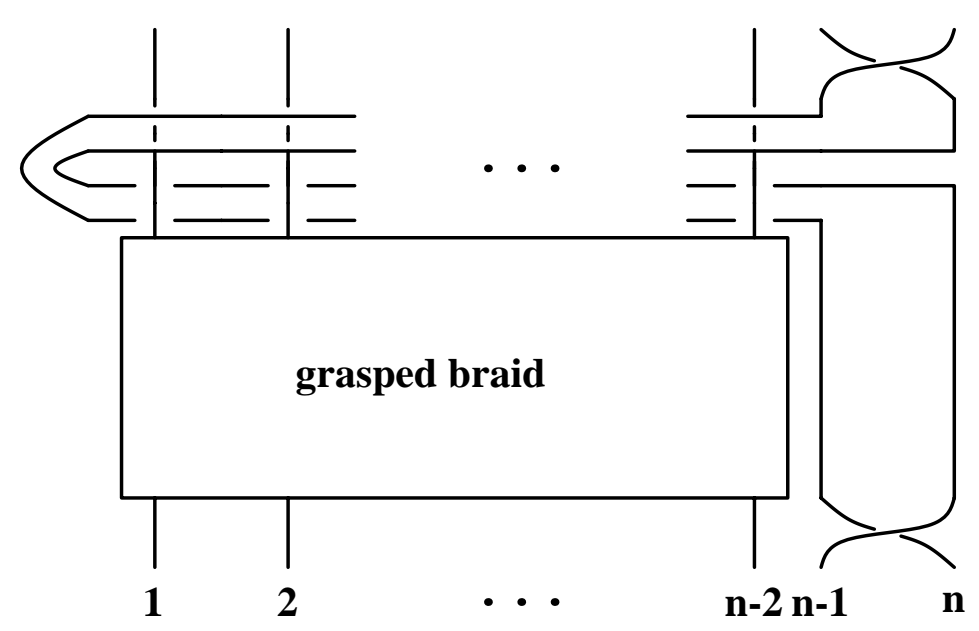

Figure 5: The double braid $\beta_{n}$ of a configuration with adjacent lines can be regarded as the grasp extension of $\beta_{n-2}$ by a $(1, \pm 1)$-cable.

Theorem 5. Let $\mathcal{L}$ be a non-singular configuration of $n \geq 3$ lines in $\mathbf{R P}^{3}$, with adjacent lines $A$ and $B$. Then,

$$
\Delta_{\mathcal{L}^{*}} \doteq\left(t_{n-1}^{l} t_{n}^{l} \prod_{i=1}^{n-2} t_{i}^{l_{i}}-1\right) \cdot F\left[\left(\Delta_{(\mathcal{L} / A=B)^{*}}\right)\right]
$$

Proof. Since for both sides of the stated identity (20), reversing of the orientation of the $i$-th line corresponds to the substitution

$$
t_{i} \rightarrow t_{i}^{-1}
$$

(up to multiplicative units), we are justified to prove the theorem for one appropriately chosen orientation. More precisely, we will adopt the conventions as discussed before the theorem, that is, we will represent $\mathcal{L}^{*}$ and $(\mathcal{L} / A=B)^{*}$ by means of $\beta_{n}$ and $\beta_{n-1}$, respectively. In this case, $l_{i}=1$ for all $1 \leq i \leq n-2$ (Equation 17). The $(i, j)$-entries of the Gassner matrices $\mu\left(\beta_{n-2}\right), \mu\left(\beta_{n-1}\right)$ and $\mu\left(\beta_{n}\right)$ will be referred to as $[i j]_{n-2},[i j]_{n-1}$ and $[i j]_{n}$, respectively. If we choose the labeled words $w_{n-2}, w_{n-1}$ and $w_{n}$ with respect to Figure 5, we have thus determined the path sets $P_{i j}^{(n-2)}, P_{i j}^{(n-1)}$ and $P_{i j}^{(n)}$. From now on, let $1 \leq i, j \leq n-2$.

- We can partition $P_{i j}^{(n)}$ as

$$
P_{i j}^{(n)}=Q_{n-2} \cup Q_{n-1} \cup Q_{n},
$$


where (see Figure 5)

$$
\begin{aligned}
Q_{n-2}= & \left\{\pi\left(s_{j n}, s_{j(n-1)}, s_{(n-1) j}, s_{n j}\right) \mid \pi \in P_{i j}^{(n-2)}\right\} \\
Q_{n-1}= & \left\{\pi\left(s_{k n}, s_{k(n-1)}, \ldots, s_{1(n-1)}, s_{(n-1) 1}, \ldots, s_{(n-1) j}, s_{n j}\right) \mid\right. \\
& \left.1 \leq k \leq n-2, \pi \in P_{i k}^{(n-2)}\right\} \\
Q_{n}= & \left\{\pi\left(s_{k n}, \ldots, s_{1 n}, s_{n 1}, \ldots, s_{n j}\right) \mid 1 \leq k \leq n-2, \pi \in P_{i k}^{(n-2)}\right\} .
\end{aligned}
$$

Therefore,

$$
\begin{aligned}
{[i j]_{n}=} & {[i j]_{n-2} t_{n-1} t_{n}+\sum_{k=1}^{n-2}[i k]_{n-2} t_{n}\left(1-t_{k}\right) t_{1} \cdots t_{j-1}\left(1-t_{n-1}\right) } \\
& +\sum_{k=1}^{n-2}[i k]_{n-2}\left(1-t_{k}\right) t_{1} \cdots t_{j-1}\left(1-t_{n}\right) \\
= & {[i j]_{n-2} t_{n-1} t_{n}+\sum_{k=1}^{n-2}[i k]_{n-2}\left(1-t_{k}\right) t_{1} \cdots t_{j-1}\left(1-t_{n-1} t_{n}\right) . }
\end{aligned}
$$

On the other hand, because $\beta_{n-1}=\beta_{n-2}^{g}$, we can apply Theorem 3:

$$
[i j]_{n-1}=[i j]_{n-2} t_{n-1}+\sum_{k=1}^{n-2}[i k]_{n-2}\left(1-t_{k}\right) t_{1} \cdots t_{j-1}\left(1-t_{n-1}\right),
$$

or, $[i j]_{n}=F\left([i j]_{n-1}\right)$.

- Since $P_{n j}^{(n)}$ consists of only one path $\pi$, which is determined by $T(\pi)=$ $\left\{s_{n j}\right\}$, we find that

$$
[n j]_{n}=t_{1} \cdots t_{j-1}\left(1-t_{n}\right) .
$$

- In order to compute $P_{i n}^{(n)}$, we have to distinguish between the values $l=-1$ and $l=1$ for link $\left(A^{*}, B^{*}\right)$. However, the corresponding entry in $\mu\left(\beta_{n}\right)$ is the same for both cases. We assume $l=1$, and leave the case $l=-1$ to the reader. Because

$$
P_{i n}^{(n)}=\left\{\pi\left(s_{k n}, \ldots, s_{1 n}, s_{n 1}, \ldots, s_{n(n-1)}\right) \mid 1 \leq k \leq n-2, \pi \in P_{i k}^{(n-2)}\right\},
$$

we see that

$$
[i n]_{n}=\sum_{k=1}^{n-2}\left(1-t_{k}\right) t_{1} \cdots t_{n-1} .
$$

- Again, we find the same value for $[i(n-1)]_{n}$ in both cases for $l$, and again we only show the computations for $l=1$. A path in $P_{i(n-1)}^{(n)}$ is either one of both types:

$$
\begin{aligned}
& \pi_{1}=\pi\left(s_{k n}, \ldots, s_{1 n}, s_{n 1}, \ldots, s_{n(n-1)}\right) \\
& \pi_{2}=\pi\left(s_{k n}, s_{k(n-1)}, \ldots, s_{1(n-1)}, s_{(n-1) 1}, \ldots, s_{n(n-1)}\right)
\end{aligned}
$$


where $k$ runs from 1 to $n-2$, and where $\pi$ is an arbitrary path in $P_{i k}^{(n-2)}$. Notice that

$$
\begin{aligned}
& T\left(\pi_{1}\right)=T(\pi) \cup\left\{s_{k n}, s_{n(n-1)}\right\} \\
& T\left(\pi_{2}\right)=T(\pi) \cup\left\{s_{k(n-1)}\right\}
\end{aligned}
$$

which yields

$$
\begin{aligned}
{[i(n-1)]_{n}=} & \sum_{k=1}^{n-2}[i k]_{n-2}\left(1-t_{k}\right) t_{1} \cdots t_{n-2}\left(1-t_{n}\right) \\
& +\sum_{k=1}^{n-2}[i k]_{n-2} t_{n}\left(1-t_{k}\right) t_{1} \cdots t_{n-2} \\
= & \sum_{k=1}^{n-2}[i k]_{n-2}\left(1-t_{k}\right) t_{1} \cdots t_{n-2} .
\end{aligned}
$$

In combination with Theorem 3 this means that

$$
[i(n-1)]_{n}=[i(n-1)]_{n-1}=F\left([i(n-1)]_{n-1}\right),
$$

the last equality following from the observation that $t_{n-1}$ does not occur $[i(n-1)]_{n-1}$.

- Similarly, as well for $l=-1$ as for $l=1$, we find that

$$
[(n-1) j]_{n}=t_{1}-t_{j-1}\left(1-t_{n-1}\right) \text {. }
$$

For example, if $l=1, P_{(n-1) j}^{(n)}$ is a pair $\left\{\pi_{1}, \pi_{2}\right\}$, with $T\left(\pi_{1}\right)=\left\{s_{(n-1) j}\right\}$ and $T\left(\pi_{2}\right)=\left\{s_{(n-1) n}, s_{n j}\right\}$.

- Using straightforward "path computations", we obtain in the case $l=$ 1:

$$
\begin{aligned}
{[(n-1)(n-1)]_{n} } & =t_{1} \cdots t_{n-2}\left(1-t_{n-1}+t_{n-1} t_{n}\right) \\
{[(n-1) n]_{n} } & =t_{1} \cdots t_{n-1}\left(1-t_{n-1}\right) \\
{[n(n-1)]_{n} } & =t_{1} \cdots t_{n-2}\left(1-t_{n}\right) \\
{[n n]_{n} } & =t_{1} \cdots t_{n-1} .
\end{aligned}
$$

- On the other hand, in the case $l=-1$ we get:

$$
\begin{aligned}
{[(n-1)(n-1)]_{n} } & =t_{1} \cdots t_{n-2} t_{n}^{-1} \\
{[(n-1) n]_{n} } & =t_{1} \cdots t_{n-2} t_{n}^{-1}\left(t_{n-1}-1\right) \\
{[n(n-1)]_{n} } & =t_{1} \cdots t_{n-2} t_{n-1}^{-1}\left(1-t_{n}^{-1}\right) \\
{[n n]_{n} } & =t_{1} \cdots t_{n-2}\left(1-t_{n}^{-1}+t_{n-1}^{-1} t_{n}^{-1}\right) .
\end{aligned}
$$


An Alexander matrix for $\mathcal{L}^{*}$ is obtained by deleting the last row of $A\left(\beta_{n}\right)-$ $I_{n}$, where $A\left(\beta_{n}\right)=\tau\left(\beta_{n}\right)=\mu\left(\beta_{n}\right)$, resulting into

$$
M=\left(\frac{A}{B}|B|\right.
$$

with $A$ an $(n-2)$ by $(n-1)$ matrix, $B$ a column of length $n-2, E$ a row of length $n-2$, and $C-1$ and $D$ single entries. From above it follows that $A$ can be obtained from $F\left(\mu\left(\beta_{n-1}\right)-I_{n-1}\right)$ by deleting the last row. Consequently, if $P^{\prime}$ denotes the minor of $A$ that is complementary to the $(n-2)$-th column,

$$
\left(t_{n-2}-1\right) \cdot P^{\prime} \doteq F\left(\Delta_{(\mathcal{L} / A=B)^{*}}\right) .
$$

On the other hand, if $P$ is the minor of $M$ complementary to the $(n-2)$-th column,

$$
\left(t_{n-2}-1\right) \cdot P \doteq \Delta_{\mathcal{L}^{*}}
$$

If $K$ denotes the last column of $A$, the previous computations of the entries of $\mu\left(\beta_{n}\right)$ imply that $B=t_{n-1} \cdot K$. This enables us to compute $P$ by subtracting $t_{n-1}$ times the $(n-1)$-th column from the $n$-th column. In other words,

$$
P= \pm\left(D-t_{n-1}(C-1)\right) \cdot P^{\prime}
$$

So, let us distinguish two cases:

- If $l=-1$ :

$$
\begin{aligned}
& D-t_{n-1}(C-1) \\
& =t_{1} \cdots t_{n-1} t_{n}^{-1}-t_{1} \cdots t_{n-2} t_{n}^{-1}-t_{n-1}\left(t_{1} \cdots t_{n-2} t_{n}^{-1}-1\right) \\
& =t_{n-1}\left(1-t_{1} \cdots t_{n-2} t_{n-1}^{-1} t_{n}^{-1}\right)
\end{aligned}
$$

- If $l=1$ :

$$
\begin{aligned}
& D-t_{n-1}(C-1) \\
& =t_{1} \cdots t_{n-1}-t_{1} \cdots t_{n-2} t_{n-1}^{2}-t_{n-1}\left(t_{1} \cdots t_{n-2}\left(1-t_{n-1}+t_{n-1} t_{n}\right)-1\right) \\
& =t_{n-1}\left(1-t_{1} \cdots t_{n}\right) .
\end{aligned}
$$

We see that in both cases,

$$
D-t_{n-1}(C-1) \doteq\left(1-t_{1} \cdots t_{n-2} t_{n-1}^{l} t_{n}^{l}\right) .
$$

We conclude:

$$
\begin{aligned}
\Delta_{\mathcal{L}^{*}} & \doteq\left(t_{n-2}-1\right) P \\
& \doteq\left(t_{n-2}-1\right)\left(1-t_{1} \cdots t_{n-2} t_{n-1}^{l} t_{n}^{l}\right) P^{\prime} \\
& \doteq\left(1-t_{1} \cdots t_{n-2} t_{n-1}^{l} t_{n}^{l}\right) F\left(\Delta_{(\mathcal{L} / A=B)^{*}}\right)
\end{aligned}
$$


In [16] the concept of "derived configuration" has been introduced as well. The relation "...is adjacent with..." is clearly an equivalence relation on a non-singular, ordered configuration $\mathcal{L}$ of $n$ lines in $\mathbf{R} \mathbf{P}^{3}$, invariant under rigid isotopies. The involved equivalence classes are called adjacency classes. The derived configuration $\mathcal{L}^{\prime}$ is in a sense the "quotient" of $\mathcal{L}$ under this equivalence relation; it is the subconfiguration obtained by taking exactly one line in each adjacency class. Notice that this is well defined up to rigid isotopy. Of course, Theorem 5 can be used to express the Alexander polynomial of a line configuration in terms of the Alexander polynomial of its derived configuration. In order to state this in a precise form, we need a few more conventions.

Suppose we can partition $\mathcal{L}$ into $p$ adjacency classes:

$$
\left\{L_{1}, \ldots, L_{k_{1}}\right\}, \ldots,\left\{L_{k_{p-1}+1}, \ldots, L_{k_{p}}\right\}
$$

with $k_{p}=n$, then we can always choose parallel orientations for the lines in the same class, that is, they match as oriented lines in the limit configuration. This choice implies that they have identical linking numbers w.r.t. the lines in another class. Furthermore, if an adjacency class is not a singleton, there is a fixed sign $\varepsilon \in\{-1,1\}$ such that for each pair $\left\{L_{i}^{*}, L_{j}^{*}\right\}$ in this class link $\left(L_{i}^{*}, L_{j}^{*}\right)=\varepsilon$. We call $\varepsilon$ the sign of a class, which is well defined, since the requirement of parallelism determines the orientations of a class up to global reversal. In the sequel we will also assume that the derived configuration $\mathcal{L}^{\prime}$ inherits its orientation from the previous choices:

$$
\left(\mathcal{L}^{\prime}\right)^{*}=\left\{L_{k_{1}}^{*}, L_{k_{2}}^{*}, \ldots, L_{k_{p}}^{*}\right\}=\left\{K_{1}^{*}, \ldots, K_{p}^{*}\right\} .
$$

Once again, we introduce an "iterator":

$$
\begin{aligned}
F: \mathbf{Z}\left[t_{1}^{ \pm 1}, \ldots, t_{p}^{ \pm 1}\right] & \rightarrow \mathbf{Z}\left[t_{1}^{ \pm 1}, \ldots, t_{n}^{ \pm 1}\right] \\
t_{1} & \rightarrow t_{1} \cdots t_{k_{1}} \\
t_{2} & \rightarrow t_{k_{1}+1} \cdots t_{k_{2}} \\
& \vdots \\
t_{p} & \rightarrow t_{k_{p-1}+1} \cdots t_{k_{p}} .
\end{aligned}
$$

Finally, for each $i=1, \ldots p$ (that is, for each adjacency class) we let $\varphi_{i}$ denote the corresponding factor:

- If the $i$-th class is a singleton then just $\varphi_{i}=1$.

- Otherwise, if $\varepsilon_{i}$ is the sign of the $i$-th class then

$$
\varphi_{i}=F\left(t_{i}^{\varepsilon_{i}} \prod_{\operatorname{link}\left(K_{i}^{*}, K_{j}^{*}\right)=1} t_{j}-\prod_{\operatorname{link}\left(K_{i}^{*}, K_{j}^{*}\right)=-1} t_{j}\right) .
$$


Corollary 6. With the previous conventions and notations, if a line configuration $\mathcal{L}$ has $p \geq 2$ adjacency classes,

$$
\Delta_{\mathcal{L}^{*}} \doteq \prod_{i=1}^{p} \varphi_{i}^{\left(k_{i}-k_{i-1}-1\right)} \cdot F\left(\Delta_{\left(\mathcal{L}^{\prime}\right)^{*}}\right) .
$$

In the case $p=1$ (trivial configuration),

$$
\Delta_{\mathcal{L}^{*}} \doteq\left(1-t_{1} \cdots t_{n}\right)^{n-2}
$$

Example. In Figure 6 the ordered configuration $\mathcal{L}$ of six oriented lines is given, as well as its derived configuration $\mathcal{L}^{\prime}=\left\{K_{1}, K_{2}, K_{3}\right\}$.

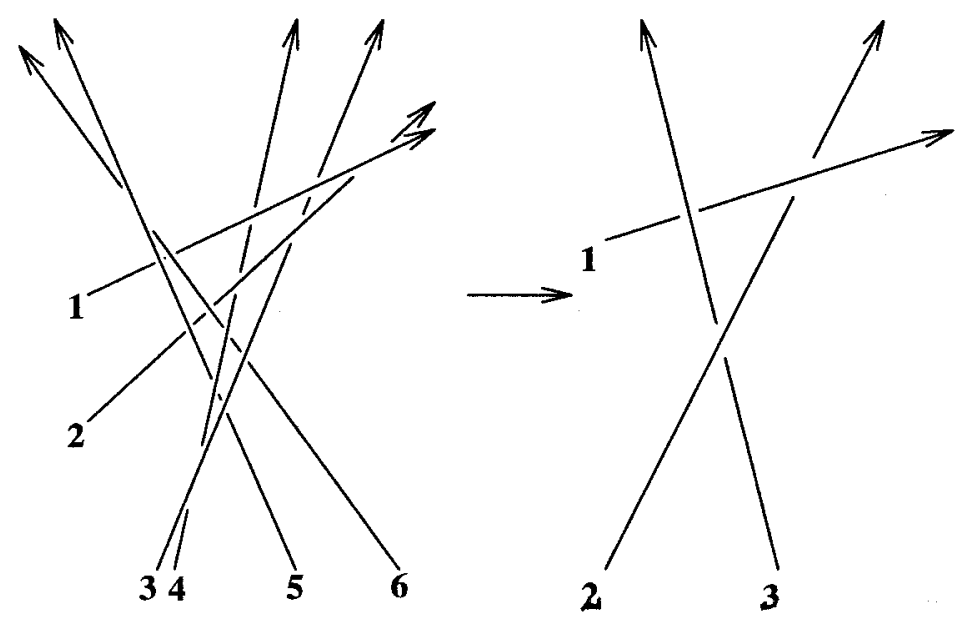

Figure 6: A configuration with three pairs of adjacent lines, resulting in a derived configuration with three lines.

The orientations are chosen according to the previous convention. The adjacency classes are $\left\{L_{1}, L_{2}\right\},\left\{L_{3}, L_{4}\right\}$ and $\left\{L_{5}, L_{6}\right\}\left(k_{1}=2, k_{2}=4\right.$, and $\left.k_{3}=6\right)$. Further, we see that the signs of these adjacency classes are all positive: $\varepsilon_{1}=\varepsilon_{2}=\varepsilon_{3}=+1$. The linking numbers in the derived configuration are:

$$
\begin{aligned}
\operatorname{link}\left(K_{1}^{*}, K_{2}^{*}\right)= & \operatorname{link}\left(K_{2}^{*}, K_{3}^{*}\right)=+1 \\
& \operatorname{link}\left(K_{1}^{*}, K_{3}^{*}\right)=-1 .
\end{aligned}
$$


Now, we can compute:

$$
\begin{aligned}
F\left(\Delta_{\mathcal{L}^{\prime}}\right) \doteq F\left(t_{2}-t_{1} t_{3}\right) & =t_{3} t_{4}-t_{1} t_{2} t_{5} t_{6} \\
\varphi_{1}=F\left(t_{1} t_{2}-t_{3}\right) & =t_{1} t_{2} t_{3} t_{4}-t_{5} t_{6} \\
\varphi_{2}=F\left(t_{1} t_{2} t_{3}-1\right) & =t_{1} t_{2} t_{3} t_{4} t_{5} t_{6}-1 \\
\varphi_{3}=F\left(t_{2} t_{3}-t_{1}\right) & =t_{3} t_{4} t_{5} t_{6}-t_{1} t_{2} .
\end{aligned}
$$

We conclude

$$
\Delta_{\mathcal{L}}=\left(t_{3} t_{4}-t_{1} t_{2} t_{5} t_{6}\right)\left(t_{1} t_{2} t_{3} t_{4}-t_{5} t_{6}\right)\left(t_{1} t_{2} t_{3} t_{4} t_{5} t_{6}-1\right)\left(t_{3} t_{4} t_{5} t_{6}-t_{1} t_{2}\right) .
$$

\section{Appendix: Alexander polynomials for configurations up to six lines.}

Below, we present all rigid isotopy types of non-singular configurations with $n$ lines, $3 \leq n \leq 6$, up to taking mirror images. Their multi-variable Alexander polynomial was computed by means of Mathematica for the indicated labels and orientations. Observe how adjacency of lines implies the appearance of simple factors in $\Delta_{\mathcal{L}}$.

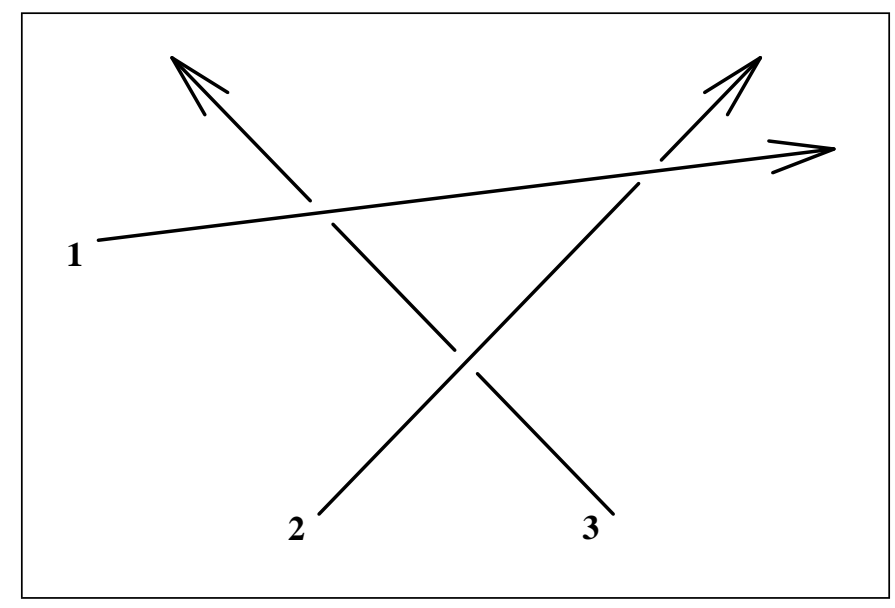

$$
\Delta=t_{1} t_{2} t_{3}-1
$$




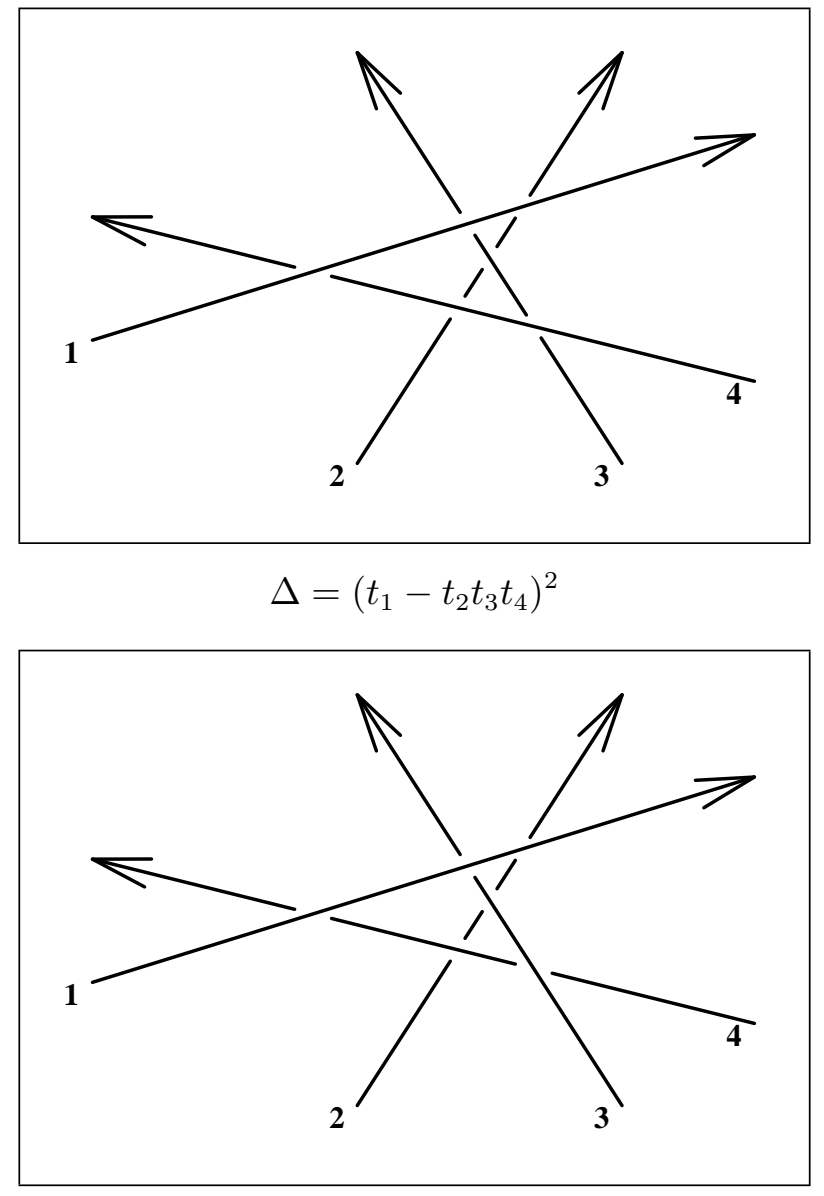

$$
\Delta=\left(t_{2}-t_{1} t_{3} t_{4}\right)\left(t_{1}-t_{2} t_{3} t_{4}\right)
$$

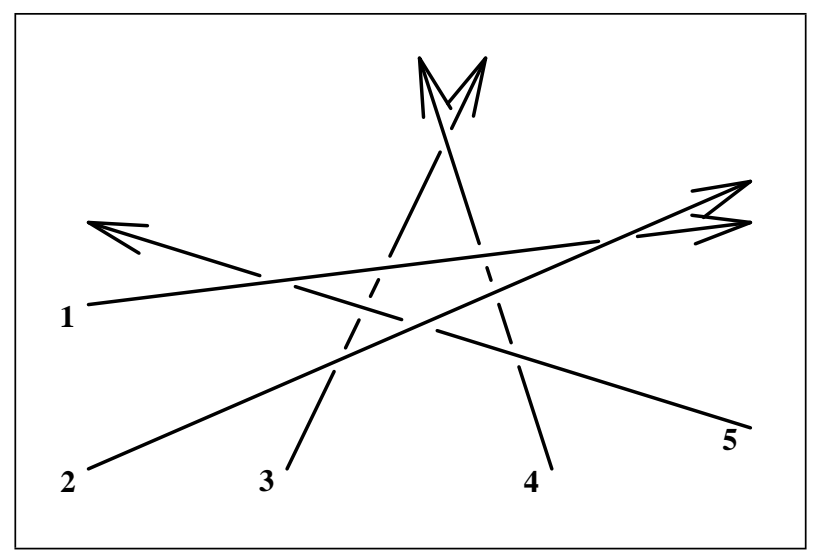

$$
\left(t_{1} t_{2}-t_{3} t_{4} t_{5}\right)^{3}
$$



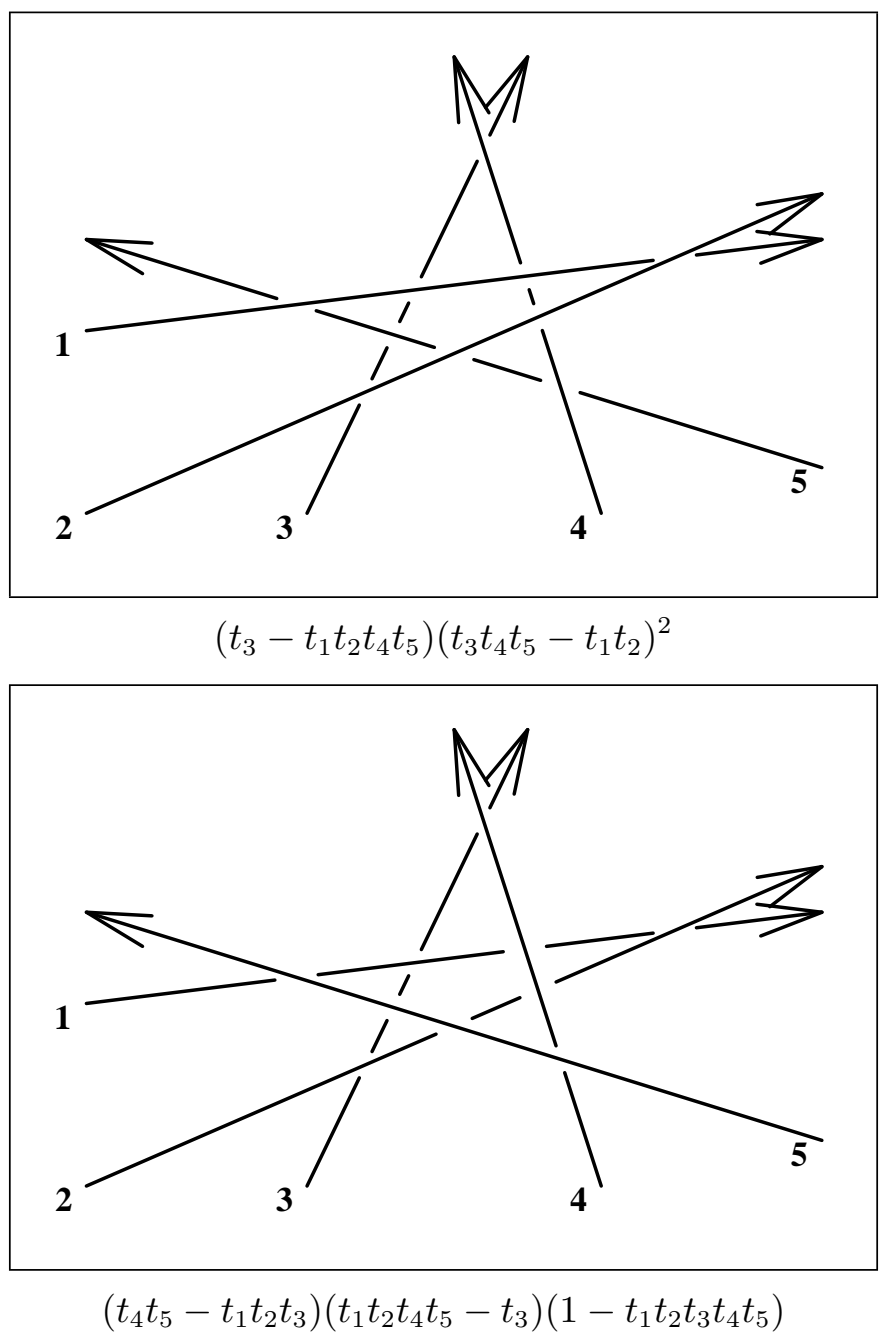


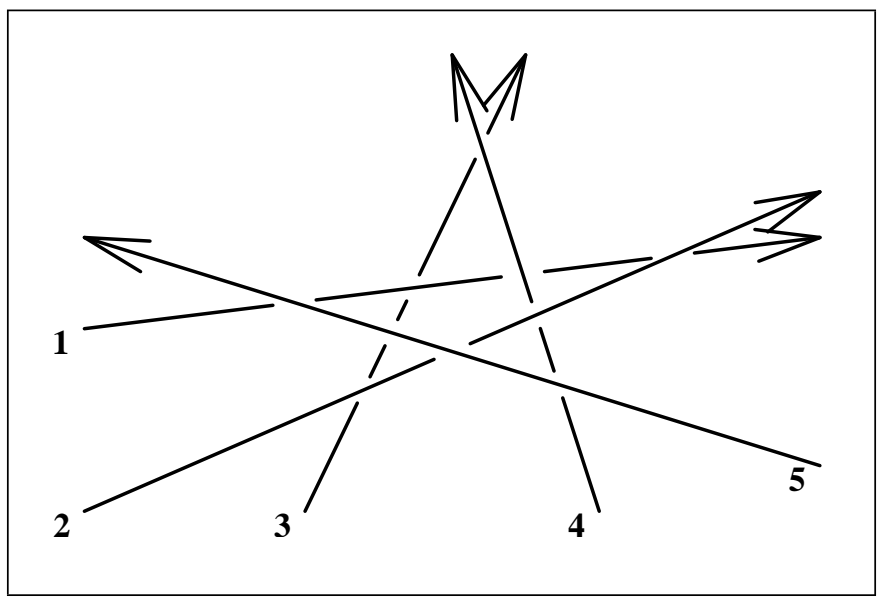

$-t_{1} t_{2}^{2} t_{3}^{2} t_{4}+t_{1}^{2} t_{2} t_{3} t_{5}+2 t_{1} t_{2} t_{3} t_{4} t_{5}-2 t_{1}^{2} t_{2} t_{3} t_{4} t_{5}-2 t_{1} t_{2}^{2} t_{3} t_{4} t_{5}+2 t_{1}^{2} t_{2}^{2} t_{3} t_{4} t_{5}-$ $2 t_{1} t_{2} t_{3}^{2} t_{4} t_{5}+2 t_{1}^{2} t_{2} t_{3}^{2} t_{4} t_{5}+2 t_{1} t_{2}^{2} t_{3}^{2} t_{4} t_{5}-2 t_{1}^{2} t_{2}^{2} t_{3}^{2} t_{4} t_{5}+t_{2} t_{3} t_{4}^{2} t_{5}-2 t_{1} t_{2} t_{3} t_{4}^{2} t_{5}+$ $t_{1}^{2} t_{2} t_{3} t_{4}^{2} t_{5}+2 t_{1} t_{2}^{2} t_{3} t_{4}^{2} t_{5}-2 t_{1}^{2} t_{2}^{2} t_{3} t_{4}^{2} t_{5}+t_{1}^{2} t_{2}^{3} t_{3} t_{4}^{2} t_{5}+2 t_{1} t_{2} t_{3}^{2} t_{4}^{2} t_{5}-2 t_{1}^{2} t_{2} t_{3}^{2} t_{4}^{2} t_{5}-$ $2 t_{1} t_{2}^{2} t_{3}^{2} t_{4}^{2} t_{5}+2 t_{1}^{2} t_{2}^{2} t_{3}^{2} t_{4}^{2} t_{5}+t_{1}^{2} t_{2} t_{3}^{3} t_{4}^{2} t_{5}-t_{1} t_{2}^{2} t_{4} t_{5}^{2}-2 t_{1} t_{2} t_{3} t_{4} t_{5}^{2}+2 t_{1}^{2} t_{2} t_{3} t_{4} t_{5}^{2}+$ $2 t_{1} t_{2}^{2} t_{3} t_{4} t_{5}^{2}-2 t_{1}^{2} t_{2}^{2} t_{3} t_{4} t_{5}^{2}-t_{1} t_{3}^{2} t_{4} t_{5}^{2}+2 t_{1} t_{2} t_{3}^{2} t_{4} t_{5}^{2}-2 t_{1}^{2} t_{2} t_{3}^{2} t_{4} t_{5}^{2}-t_{1} t_{2}^{2} t_{3}^{2} t_{4} t_{5}^{2}+$ $2 t_{1}^{2} t_{2}^{2} t_{3}^{2} t_{4} t_{5}^{2}-t_{1}^{3} t_{2}^{2} t_{3}^{2} t_{4} t_{5}^{2}+2 t_{1} t_{2} t_{3} t_{4}^{2} t_{5}^{2}-2 t_{1}^{2} t_{2} t_{3} t_{4}^{2} t_{5}^{2}-2 t_{1} t_{2}^{2} t_{3} t_{4}^{2} t_{5}^{2}+2 t_{1}^{2} t_{2}^{2} t_{3} t_{4}^{2} t_{5}^{2}-$ $2 t_{1} t_{2} t_{3}^{2} t_{4}^{2} t_{5}^{2}+2 t_{1}^{2} t_{2} t_{3}^{2} t_{4}^{2} t_{5}^{2}+2 t_{1} t_{2}^{2} t_{3}^{2} t_{4}^{2} t_{5}^{2}-2 t_{1}^{2} t_{2}^{2} t_{3}^{2} t_{4}^{2} t_{5}^{2}-t_{1} t_{2}^{2} t_{3}^{2} t_{4}^{3} t_{5}^{2}+t_{1}^{2} t_{2} t_{3} t_{4}^{2} t_{5}^{3}$

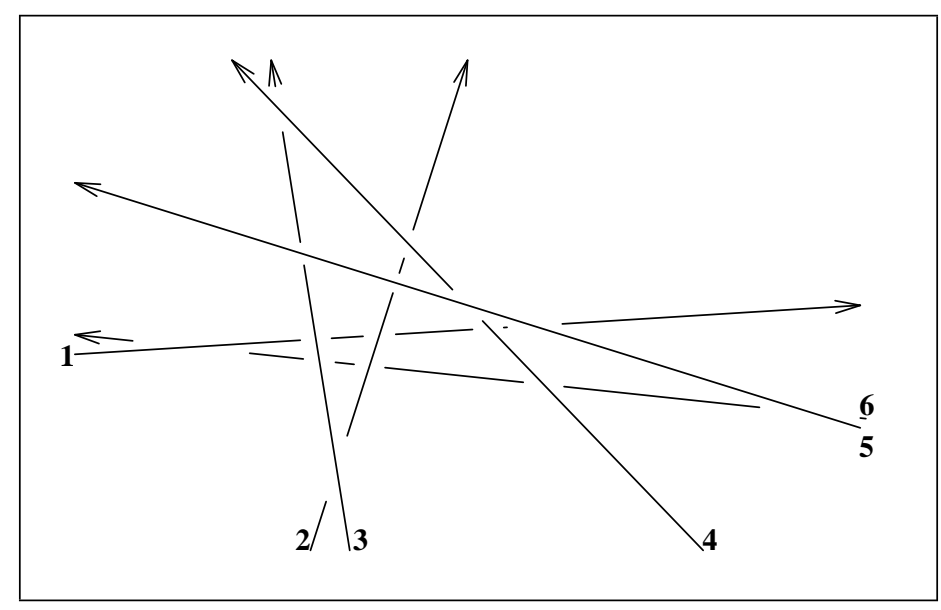

$\left(t_{1} t_{2} t_{3} t_{4} t_{5}-t_{6}\right)^{4}$ 


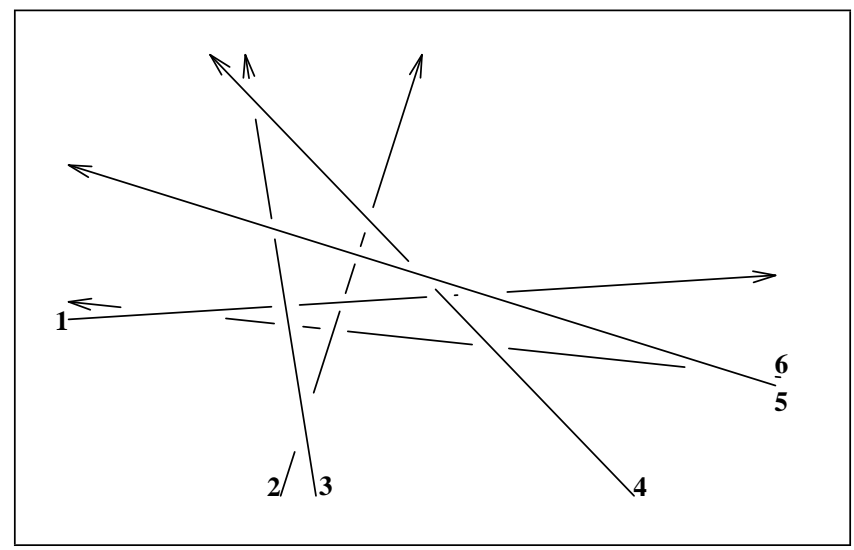

$\left(t_{1} t_{2} t_{3} t_{4} t_{5}-t_{6}\right)^{3}\left(t_{3} t_{4} t_{5}-t_{1} t_{2} t_{6}\right)$

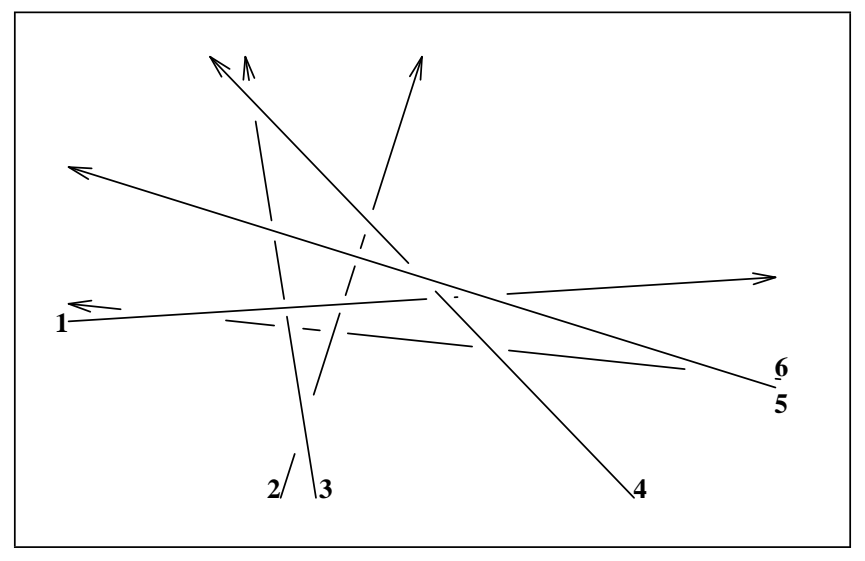

$$
\left(t_{1} t_{2} t_{3} t_{4} t_{5}-t_{6}\right)^{2}\left(t_{2} t_{3} t_{4} t_{5}-t_{1} t_{6}\right)\left(t_{4} t_{5}-t_{1} t_{2} t_{3} t_{6}\right)
$$

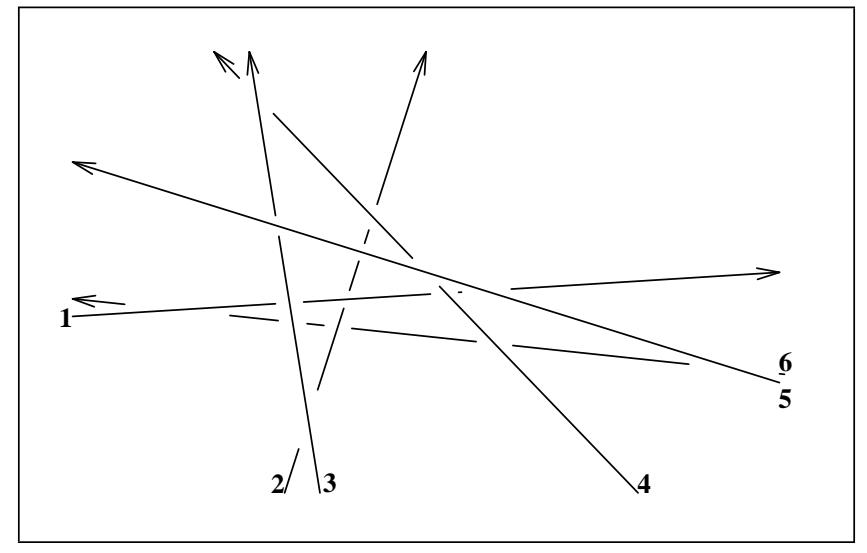

$\left(t_{1} t_{2} t_{3} t_{4} t_{5}-t_{6}\right)^{2}\left(t_{3} t_{4} t_{5}-t_{1} t_{2} t_{6}\right)\left(t_{1} t_{2} t_{5}-t_{3} t_{4} t_{6}\right)$ 


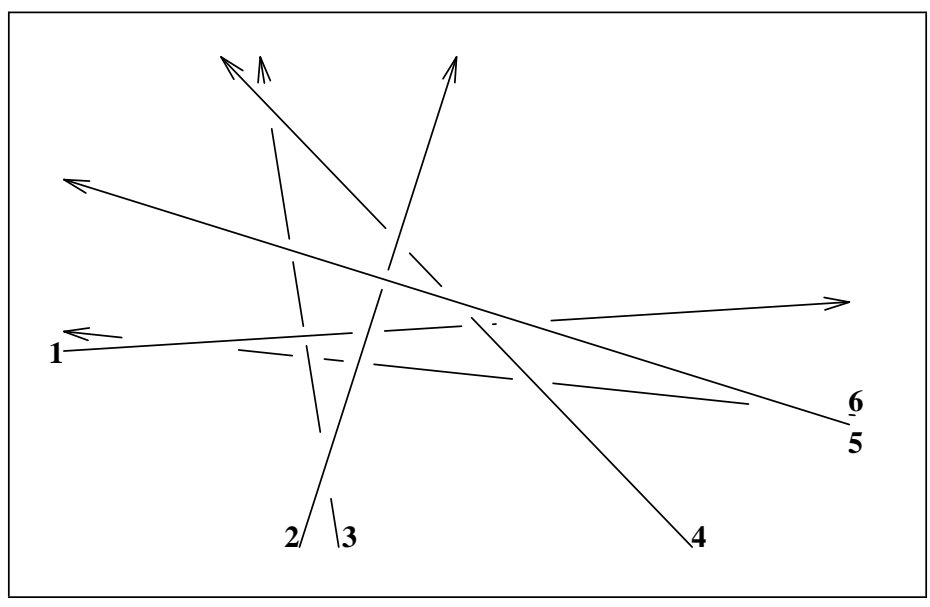

$\left(t_{1} t_{2} t_{3} t_{4} t_{5}-t_{6}\right)\left(t_{1}^{2} t_{2} t_{3} t_{4}^{2} t_{5}^{3}-t_{1} t_{2}^{2} t_{4} t_{5}^{2} t_{6}-2 t_{1} t_{2} t_{3} t_{4} t_{5}^{2} t_{6}+2 t_{1}^{2} t_{2} t_{3} t_{4} t_{5}^{2} t_{6}+2 t_{1} t_{2}^{2} t_{3} t_{4} t_{5}^{2} t_{6}-\right.$ $2 t_{1}^{2} t_{2}^{2} t_{3} t_{4} t_{5}^{2} t_{6}-t_{1} t_{3}^{2} t_{4} t_{5}^{2} t_{6}+2 t_{1} t_{2} t_{3}^{2} t_{4} t_{5}^{2} t_{6}-2 t_{1}^{2} t_{2} t_{3}^{2} t_{4} t_{5}^{2} t_{6}-t_{1} t_{2}^{2} t_{3}^{2} t_{4} t_{5}^{2} t_{6}+2 t_{1}^{2} t_{2}^{2} t_{3}^{2} t_{4} t_{5}^{2} t_{6}-$ $t_{1}^{3} t_{2}^{2} t_{3}^{2} t_{4} t_{5}^{2} t_{6}+2 t_{1} t_{2} t_{3} t_{4}^{2} t_{5}^{2} t_{6}-2 t_{1}^{2} t_{2} t_{3} t_{4}^{2} t_{5}^{2} t_{6}-2 t_{1} t_{2}^{2} t_{3} t_{4}^{2} t_{5}^{2} t_{6}+2 t_{1}^{2} t_{2}^{2} t_{3} t_{4}^{2} t_{5}^{2} t_{6}-2 t_{1} t_{2} t_{3}^{2} t_{4}^{2} t_{5}^{2} t_{6}+$ $2 t_{1}^{2} t_{2} t_{3}^{2} t_{4}^{2} t_{5}^{2} t_{6}+2 t_{1} t_{2}^{2} t_{3}^{2} t_{4}^{2} t_{5}^{2} t_{6}-2 t_{1}^{2} t_{2}^{2} t_{3}^{2} t_{4}^{2} t_{5}^{2} t_{6}-t_{1} t_{2}^{2} t_{3}^{2} t_{4}^{3} t_{5}^{2} t_{6}+t_{1}^{2} t_{2} t_{3} t_{5} t_{6}^{2}+2 t_{1} t_{2} t_{3} t_{4} t_{5} t_{6}^{2}-$ $2 t_{1}^{2} t_{2} t_{3} t_{4} t_{5} t_{6}^{2}-2 t_{1} t_{2}^{2} t_{3} t_{4} t_{5} t_{6}^{2}+2 t_{1}^{2} t_{2}^{2} t_{3} t_{4} t_{5} t_{6}^{2}-2 t_{1} t_{2} t_{3}^{2} t_{4} t_{5} t_{6}^{2}+2 t_{1}^{2} t_{2} t_{3}^{2} t_{4} t_{5} t_{6}^{2}+2 t_{1} t_{2}^{2} t_{3}^{2} t_{4} t_{5} t_{6}^{2}-$ $2 t_{1}^{2} t_{2}^{2} t_{3}^{2} t_{4} t_{5} t_{6}^{2}+t_{2} t_{3} t_{4}^{2} t_{5} t_{6}^{2}-2 t_{1} t_{2} t_{3} t_{4}^{2} t_{5} t_{6}^{2}+t_{1}^{2} t_{2} t_{3} t_{4}^{2} t_{5} t_{6}^{2}+2 t_{1} t_{2}^{2} t_{3} t_{4}^{2} t_{5} t_{6}^{2}-2 t_{1}^{2} t_{2}^{2} t_{3} t_{4}^{2} t_{5} t_{6}^{2}+$ $t_{1}^{2} t_{2}^{3} t_{3} t_{4}^{2} t_{5} t_{6}^{2}+2 t_{1} t_{2} t_{3}^{2} t_{4}^{2} t_{5} t_{6}^{2}-2 t_{1}^{2} t_{2} t_{3}^{2} t_{4}^{2} t_{5} t_{6}^{2}-2 t_{1} t_{2}^{2} t_{3}^{2} t_{4}^{2} t_{5} t_{6}^{2}+2 t_{1}^{2} t_{2}^{2} t_{3}^{2} t_{4}^{2} t_{5} t_{6}^{2}+t_{1}^{2} t_{2} t_{3}^{3} t_{4}^{2} t_{5} t_{6}^{2}-$ $\left.t_{1} t_{2}^{2} t_{3}^{2} t_{4} t_{6}^{3}\right)$

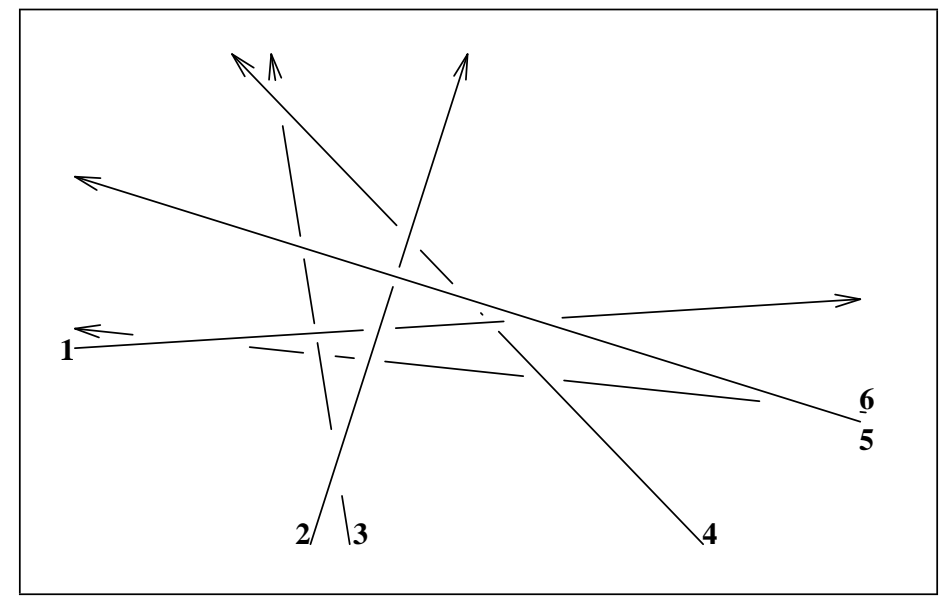

$\left(t_{1} t_{2} t_{3} t_{4} t_{5}-t_{6}\right)\left(t_{3} t_{4} t_{5}-t_{1} t_{2} t_{6}\right)\left(t_{1} t_{2} t_{5}-t_{3} t_{4} t_{6}\right)\left(t_{5}-t_{1} t_{2} t_{3} t_{4} t_{6}\right)$ 


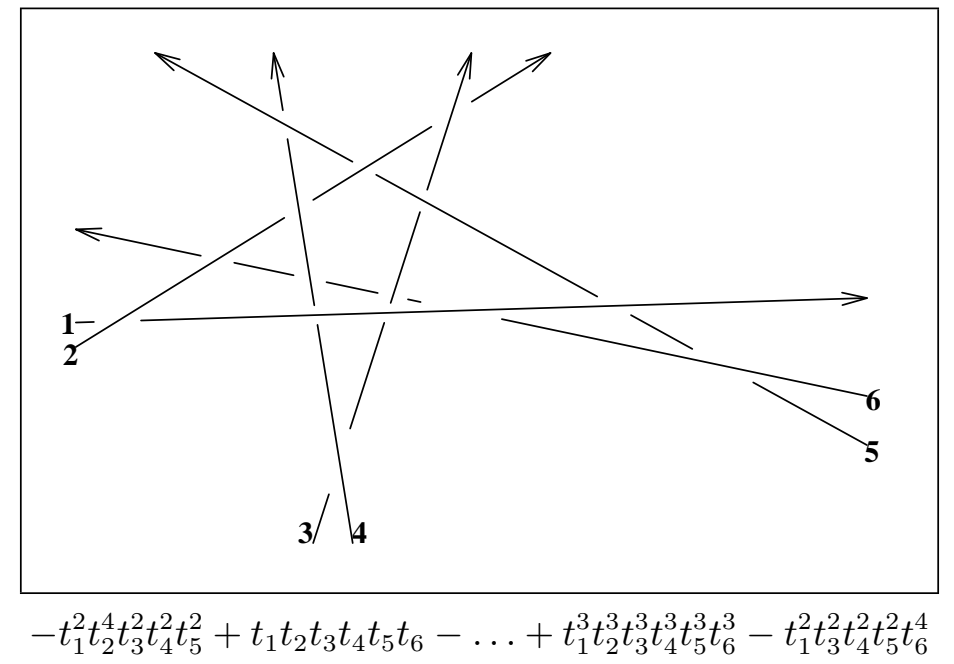

(667 terms in total)

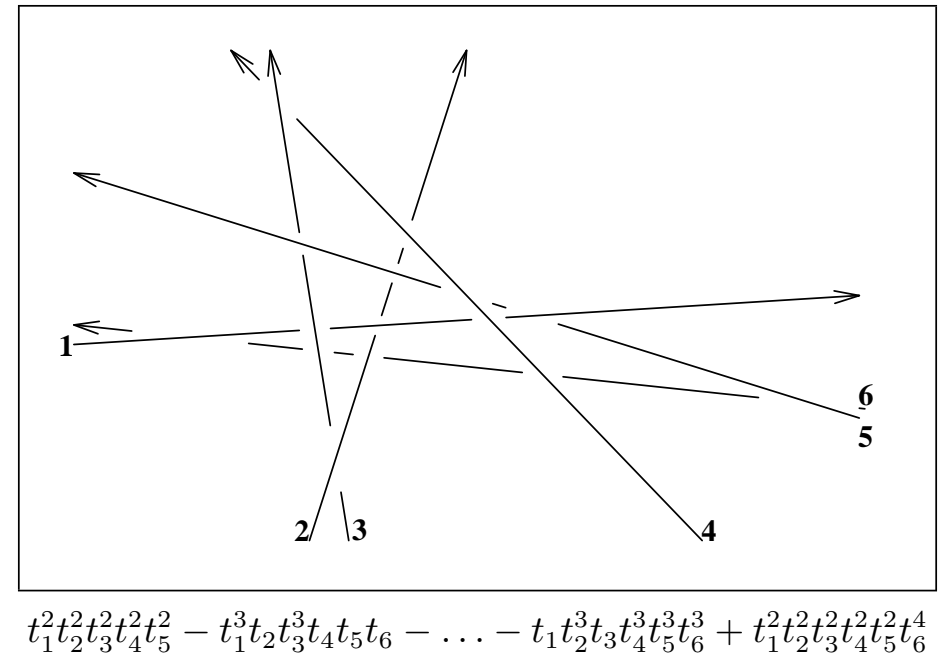

(317 terms in total) 

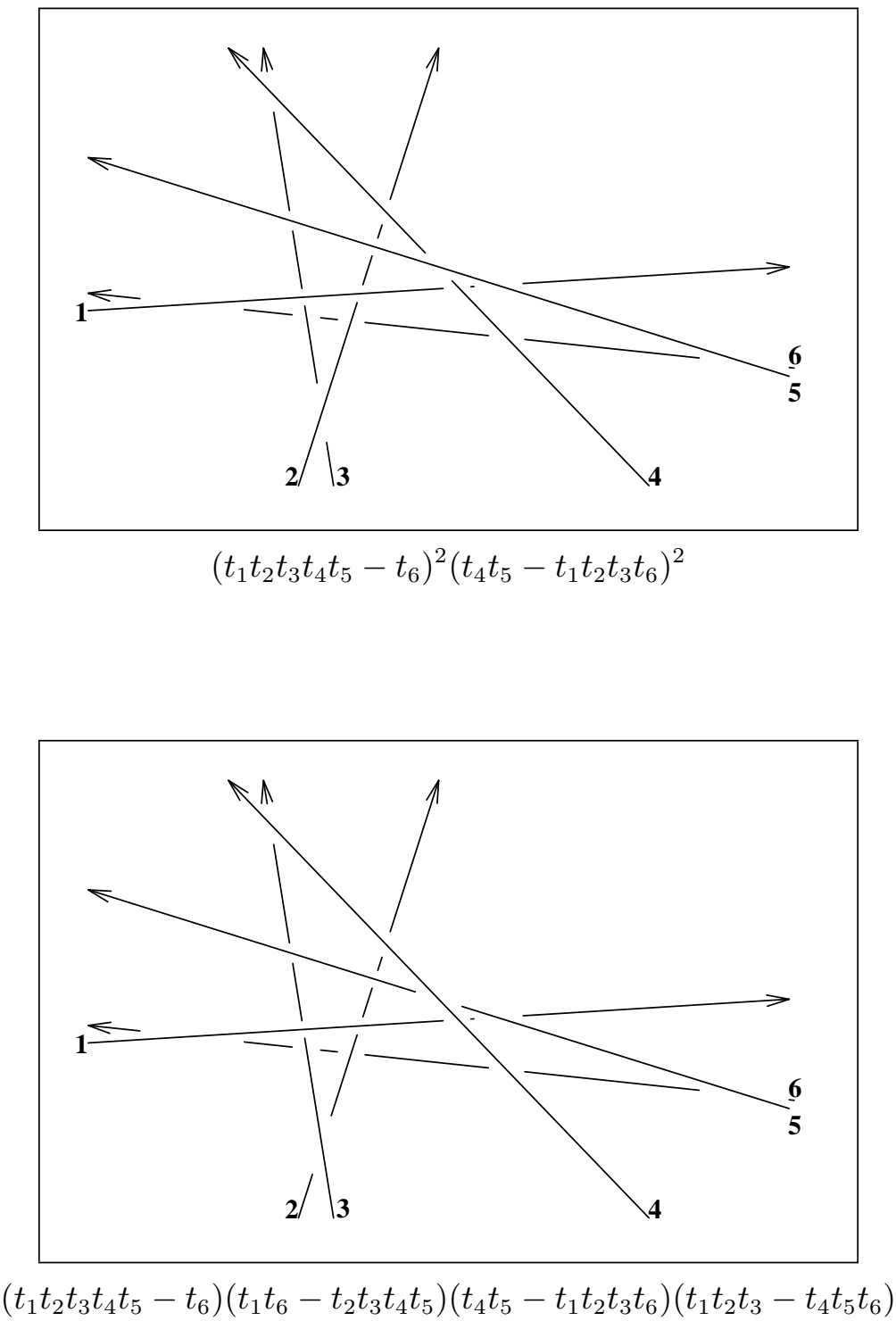


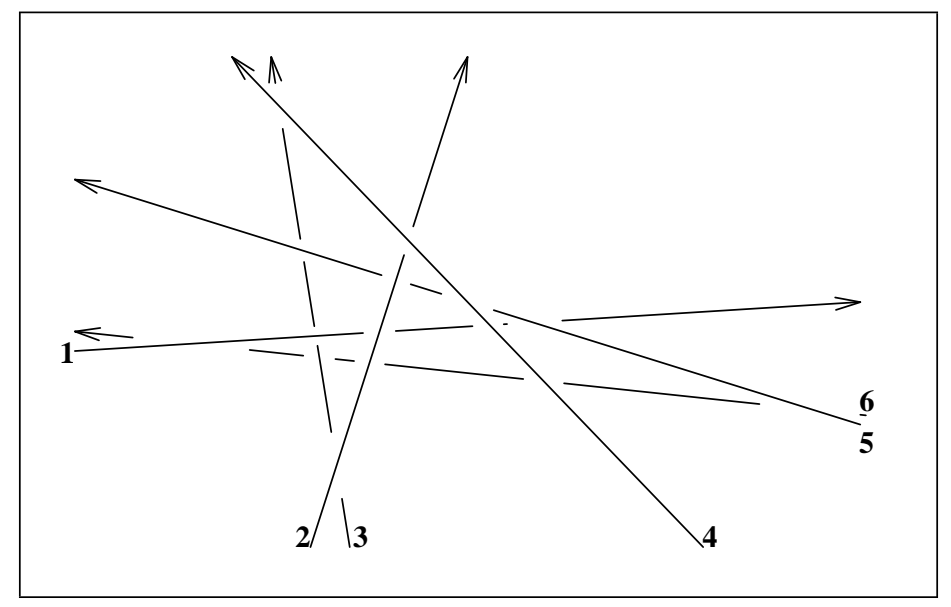

$t_{1}^{3} t_{2}^{2} t_{3}^{2} t_{4}^{3} t_{5}^{2}-t_{1}^{2} t_{2}^{3} t_{3} t_{4}^{2} t_{5} t_{6}-2 t_{1}^{2} t_{2}^{2} t_{3}^{2} t_{4}^{2} t_{5} t_{6}+2 t_{1}^{3} t_{2}^{2} t_{3}^{2} t_{4}^{2} t_{5} t_{6}+2 t_{1}^{2} t_{2}^{3} t_{3}^{2} t_{4}^{2} t_{5} t_{6}-2 t_{1}^{3} t_{2}^{3} t_{3}^{2} t_{4}^{2} t_{5} t_{6}-$ $t_{1}^{2} t_{2} t_{3}^{3} t_{4}^{2} t_{5} t_{6}+2 t_{1}^{2} t_{2}^{2} t_{3}^{3} t_{4}^{2} t_{5} t_{6}-2 t_{1}^{3} t_{2}^{2} t_{3}^{3} t_{4}^{2} t_{5} t_{6}-t_{1}^{2} t_{2}^{3} t_{3}^{3} t_{4}^{2} t_{5} t_{6}+2 t_{1}^{3} t_{2}^{3} t_{3}^{3} t_{4}^{2} t_{5} t_{6}-t_{1}^{4} t_{2}^{3} t_{3}^{3} t_{4}^{2} t_{5} t_{6}-$ $2 t_{1}^{2} t_{2}^{2} t_{3} t_{4}^{2} t_{5}^{2} t_{6}+2 t_{1}^{2} t_{2}^{3} t_{3} t_{4}^{2} t_{5}^{2} t_{6}+4 t_{1}^{2} t_{2}^{2} t_{3}^{2} t_{4}^{2} t_{5}^{2} t_{6}-2 t_{1}^{3} t_{2}^{2} t_{3}^{2} t_{4}^{2} t_{5}^{2} t_{6}-4 t_{1}^{2} t_{2}^{3} t_{3}^{2} t_{4}^{2} t_{5}^{2} t_{6}+2 t_{1}^{3} t_{2}^{3} t_{3}^{2} t_{4}^{2} t_{5}^{2} t_{6}-$ $2 t_{1}^{2} t_{2}^{2} t_{3}^{3} t_{4}^{2} t_{5}^{2} t_{6}+2 t_{1}^{3} t_{2}^{2} t_{3}^{3} t_{4}^{2} t_{5}^{2} t_{6}+2 t_{1}^{2} t_{2}^{3} t_{3}^{3} t_{4}^{2} t_{5}^{2} t_{6}-2 t_{1}^{3} t_{2}^{3} t_{3}^{3} t_{4}^{2} t_{5}^{2} t_{6}+2 t_{1}^{2} t_{2}^{2} t_{3} t_{4}^{3} t_{5}^{2} t_{6}-2 t_{1}^{2} t_{2}^{3} t_{3} t_{4}^{3} t_{5}^{2} t_{6}-$ $2 t_{1}^{2} t_{2}^{2} t_{3}^{2} t_{4}^{3} t_{5}^{2} t_{6}+2 t_{1}^{2} t_{2}^{3} t_{3}^{2} t_{4}^{3} t_{5}^{2} t_{6}-t_{1}^{2} t_{2} t_{3} t_{4}^{2} t_{5}^{3} t_{6}+2 t_{1}^{2} t_{2}^{2} t_{3} t_{4}^{2} t_{5}^{3} t_{6}-t_{1}^{2} t_{2}^{3} t_{3} t_{4}^{2} t_{5}^{3} t_{6}-2 t_{1}^{2} t_{2}^{2} t_{3}^{2} t_{4}^{2} t_{5}^{3} t_{6}+$ $2 t_{1}^{2} t_{2}^{3} t_{3}^{2} t_{4}^{2} t_{5}^{3} t_{6}-t_{1}^{2} t_{2}^{3} t_{3}^{3} t_{4}^{2} t_{5}^{3} t_{6}-2 t_{1}^{2} t_{2}^{2} t_{3} t_{4}^{3} t_{5}^{3} t_{6}+2 t_{1}^{2} t_{2}^{3} t_{3} t_{4}^{3} t_{5}^{3} t_{6}+2 t_{1}^{2} t_{2}^{2} t_{3}^{2} t_{4}^{3} t_{5}^{3} t_{6}-2 t_{1}^{2} t_{2}^{3} t_{3}^{2} t_{4}^{3} t_{5}^{3} t_{6}-$ $t_{1}^{2} t_{2}^{3} t_{3} t_{4}^{4} t_{5}^{3} t_{6}+t_{1}^{3} t_{2}^{2} t_{3}^{2} t_{4} t_{6}^{2}+2 t_{1}^{2} t_{2} t_{3}^{2} t_{4} t_{5} t_{6}^{2}-2 t_{1}^{2} t_{2}^{2} t_{3}^{2} t_{4} t_{5} t_{6}^{2}-2 t_{1}^{2} t_{2} t_{3}^{3} t_{4} t_{5} t_{6}^{2}+2 t_{1}^{2} t_{2}^{2} t_{3}^{3} t_{4} t_{5} t_{6}^{2}-$ $2 t_{1}^{2} t_{2} t_{3}^{2} t_{4}^{2} t_{5} t_{6}^{2}+4 t_{1}^{2} t_{2}^{2} t_{3}^{2} t_{4}^{2} t_{5} t_{6}^{2}-2 t_{1}^{3} t_{2}^{2} t_{3}^{2} t_{4}^{2} t_{5} t_{6}^{2}-2 t_{1}^{2} t_{2}^{3} t_{3}^{2} t_{4}^{2} t_{5} t_{6}^{2}+2 t_{1}^{3} t_{2}^{3} t_{3}^{2} t_{4}^{2} t_{5} t_{6}^{2}+2 t_{1}^{2} t_{2} t_{3}^{3} t_{4}^{2} t_{5} t_{6}^{2}-$ $4 t_{1}^{2} t_{2}^{2} t_{3}^{3} t_{4}^{2} t_{5} t_{6}^{2}+2 t_{1}^{3} t_{2}^{2} t_{3}^{3} t_{4}^{2} t_{5} t_{6}^{2}+2 t_{1}^{2} t_{2}^{3} t_{3}^{3} t_{4}^{2} t_{5} t_{6}^{2}-2 t_{1}^{3} t_{2}^{3} t_{3}^{3} t_{4}^{2} t_{5} t_{6}^{2}+t_{1} t_{3}^{2} t_{4} t_{5}^{2} t_{6}^{2}-2 t_{1}^{2} t_{2} t_{3}^{2} t_{4} t_{5}^{2} t_{6}^{2}+$ $2 t_{1}^{2} t_{2}^{2} t_{3}^{2} t_{4} t_{5}^{2} t_{6}^{2}+2 t_{1}^{2} t_{2} t_{3}^{3} t_{4} t_{5}^{2} t_{6}^{2}-2 t_{1}^{2} t_{2}^{2} t_{3}^{3} t_{4} t_{5}^{2} t_{6}^{2}+t_{1}^{3} t_{2}^{2} t_{3}^{4} t_{4} t_{5}^{2} t_{6}^{2}+2 t_{1} t_{2} t_{3} t_{4}^{2} t_{5}^{2} t_{6}^{2}-2 t_{1}^{2} t_{2} t_{3} t_{4}^{2} t_{5}^{2} t_{6}^{2}-$ $2 t_{1} t_{2}^{2} t_{3} t_{4}^{2} t_{5}^{2} t_{6}^{2}+4 t_{1}^{2} t_{2}^{2} t_{3} t_{4}^{2} t_{5}^{2} t_{6}^{2}-2 t_{1}^{2} t_{2}^{3} t_{3} t_{4}^{2} t_{5}^{2} t_{6}^{2}-2 t_{1} t_{2} t_{3}^{2} t_{4}^{2} t_{5}^{2} t_{6}^{2}+4 t_{1}^{2} t_{2} t_{3}^{2} t_{4}^{2} t_{5}^{2} t_{6}^{2}+2 t_{1} t_{2}^{2} t_{3}^{2} t_{4}^{2} t_{5}^{2} t_{6}^{2}-$ $8 t_{1}^{2} t_{2}^{2} t_{3}^{2} t_{4}^{2} t_{5}^{2} t_{6}^{2}+2 t_{1}^{3} t_{2}^{2} t_{3}^{2} t_{4}^{2} t_{5}^{2} t_{6}^{2}+4 t_{1}^{2} t_{2}^{3} t_{3}^{2} t_{4}^{2} t_{5}^{2} t_{6}^{2}-2 t_{1}^{3} t_{2}^{3} t_{3}^{2} t_{4}^{2} t_{5}^{2} t_{6}^{2}-2 t_{1}^{2} t_{2} t_{3}^{3} t_{4}^{2} t_{5}^{2} t_{6}^{2}+4 t_{1}^{2} t_{2}^{2} t_{3}^{3} t_{4}^{2} t_{5}^{2} t_{6}^{2}-$ $2 t_{1}^{3} t_{2}^{2} t_{3}^{3} t_{4}^{2} t_{5}^{2} t_{6}^{2}-2 t_{1}^{2} t_{2}^{3} t_{3}^{3} t_{4}^{2} t_{5}^{2} t_{6}^{2}+2 t_{1}^{3} t_{2}^{3} t_{3}^{3} t_{4}^{2} t_{5}^{2} t_{6}^{2}+t_{1} t_{2}^{2} t_{4}^{3} t_{5}^{2} t_{6}^{2}-2 t_{1}^{2} t_{2}^{2} t_{3} t_{4}^{3} t_{5}^{2} t_{6}^{2}+2 t_{1}^{2} t_{2}^{3} t_{3} t_{4}^{3} t_{5}^{2} t_{6}^{2}+$ $2 t_{1}^{2} t_{2}^{2} t_{3}^{2} t_{4}^{3} t_{5}^{2} t_{6}^{2}-2 t_{1}^{2} t_{2}^{3} t_{3}^{2} t_{4}^{3} t_{5}^{2} t_{6}^{2}+t_{1}^{3} t_{2}^{4} t_{3}^{2} t_{4}^{3} t_{5}^{2} t_{6}^{2}-2 t_{1} t_{2} t_{3} t_{4}^{2} t_{5}^{3} t_{6}^{2}+2 t_{1}^{2} t_{2} t_{3} t_{4}^{2} t_{5}^{3} t_{6}^{2}+2 t_{1} t_{2}^{2} t_{3} t_{4}^{2} t_{5}^{3} t_{6}^{2}-$ $4 t_{1}^{2} t_{2}^{2} t_{3} t_{4}^{2} t_{5}^{3} t_{6}^{2}+2 t_{1}^{2} t_{2}^{3} t_{3} t_{4}^{2} t_{5}^{3} t_{6}^{2}+2 t_{1} t_{2} t_{3}^{2} t_{4}^{2} t_{5}^{3} t_{6}^{2}-2 t_{1}^{2} t_{2} t_{3}^{2} t_{4}^{2} t_{5}^{3} t_{6}^{2}-2 t_{1} t_{2}^{2} t_{3}^{2} t_{4}^{2} t_{5}^{3} t_{6}^{2}+4 t_{1}^{2} t_{2}^{2} t_{3}^{2} t_{4}^{2} t_{5}^{3} t_{6}^{2}-$ $2 t_{1}^{2} t_{2}^{3} t_{3}^{2} t_{4}^{2} t_{5}^{3} t_{6}^{2}+2 t_{1}^{2} t_{2}^{2} t_{3} t_{4}^{3} t_{5}^{3} t_{6}^{2}-2 t_{1}^{2} t_{2}^{3} t_{3} t_{4}^{3} t_{5}^{3} t_{6}^{2}-2 t_{1}^{2} t_{2}^{2} t_{3}^{2} t_{4}^{3} t_{5}^{3} t_{6}^{2}+2 t_{1}^{2} t_{2}^{3} t_{3}^{2} t_{4}^{3} t_{5}^{3} t_{6}^{2}+t_{1} t_{2}^{2} t_{3}^{2} t_{4}^{3} t_{5}^{4} t_{6}^{2}-$ $t_{1}^{2} t_{2} t_{3}^{3} t_{5} t_{6}^{3}-2 t_{1}^{2} t_{2} t_{3}^{2} t_{4} t_{5} t_{6}^{3}+2 t_{1}^{2} t_{2}^{2} t_{3}^{2} t_{4} t_{5} t_{6}^{3}+2 t_{1}^{2} t_{2} t_{3}^{3} t_{4} t_{5} t_{6}^{3}-2 t_{1}^{2} t_{2}^{2} t_{3}^{3} t_{4} t_{5} t_{6}^{3}-t_{1}^{2} t_{2} t_{3} t_{4}^{2} t_{5} t_{6}^{3}+$ $2 t_{1}^{2} t_{2} t_{3}^{2} t_{4}^{2} t_{5} t_{6}^{3}-2 t_{1}^{2} t_{2}^{2} t_{3}^{2} t_{4}^{2} t_{5} t_{6}^{3}-t_{1}^{2} t_{2} t_{3}^{3} t_{4}^{2} t_{5} t_{6}^{3}+2 t_{1}^{2} t_{2}^{2} t_{3}^{3} t_{4}^{2} t_{5} t_{6}^{3}-t_{1}^{2} t_{2}^{3} t_{3}^{3} t_{4}^{2} t_{5} t_{6}^{3}+2 t_{1}^{2} t_{2} t_{3}^{2} t_{4} t_{5}^{2} t_{6}^{3}-$ $2 t_{1}^{2} t_{2}^{2} t_{3}^{2} t_{4} t_{5}^{2} t_{6}^{3}-2 t_{1}^{2} t_{2} t_{3}^{3} t_{4} t_{5}^{2} t_{6}^{3}+2 t_{1}^{2} t_{2}^{2} t_{3}^{3} t_{4} t_{5}^{2} t_{6}^{3}-2 t_{1} t_{2} t_{3} t_{4}^{2} t_{5}^{2} t_{6}^{3}+2 t_{1}^{2} t_{2} t_{3} t_{4}^{2} t_{5}^{2} t_{6}^{3}+2 t_{1} t_{2}^{2} t_{3} t_{4}^{2} t_{5}^{2} t_{6}^{3}-$ $2 t_{1}^{2} t_{2}^{2} t_{3} t_{4}^{2} t_{5}^{2} t_{6}^{3}+2 t_{1} t_{2} t_{3}^{2} t_{4}^{2} t_{5}^{2} t_{6}^{3}-4 t_{1}^{2} t_{2} t_{3}^{2} t_{4}^{2} t_{5}^{2} t_{6}^{3}-2 t_{1} t_{2}^{2} t_{3}^{2} t_{4}^{2} t_{5}^{2} t_{6}^{3}+4 t_{1}^{2} t_{2}^{2} t_{3}^{2} t_{4}^{2} t_{5}^{2} t_{6}^{3}+2 t_{1}^{2} t_{2} t_{3}^{3} t_{4}^{2} t_{5}^{2} t_{6}^{3}-$ $2 t_{1}^{2} t_{2}^{2} t_{3}^{3} t_{4}^{2} t_{5}^{2} t_{6}^{3}-t_{2} t_{3} t_{4}^{2} t_{5}^{3} t_{6}^{3}+2 t_{1} t_{2} t_{3} t_{4}^{2} t_{5}^{3} t_{6}^{3}-t_{1}^{2} t_{2} t_{3} t_{4}^{2} t_{5}^{3} t_{6}^{3}-2 t_{1} t_{2}^{2} t_{3} t_{4}^{2} t_{5}^{3} t_{6}^{3}+2 t_{1}^{2} t_{2}^{2} t_{3} t_{4}^{2} t_{5}^{3} t_{6}^{3}-$ $t_{1}^{2} t_{2}^{3} t_{3} t_{4}^{2} t_{5}^{3} t_{6}^{3}-2 t_{1} t_{2} t_{3}^{2} t_{4}^{2} t_{5}^{3} t_{6}^{3}+2 t_{1}^{2} t_{2} t_{3}^{2} t_{4}^{2} t_{5}^{3} t_{6}^{3}+2 t_{1} t_{2}^{2} t_{3}^{2} t_{4}^{2} t_{5}^{3} t_{6}^{3}-2 t_{1}^{2} t_{2}^{2} t_{3}^{2} t_{4}^{2} t_{5}^{3} t_{6}^{3}-t_{1}^{2} t_{2} t_{3}^{3} t_{4}^{2} t_{5}^{3} t_{6}^{3}+$ $t_{1} t_{2}^{2} t_{3}^{2} t_{4} t_{5}^{2} t_{6}^{4}$

\section{References}

[1] J.W. Alexander, Topological invariants of knots and links, Trans. Amer. Math. Soc., 30 (1928), 275-306.

[2] E. Artin, The theory of braids, Ann. of Math., 48 (1947), 101-126. 
[3] J.S. Birman, Braids, Links, and Mapping Class Groups, Annals of Mathematics Studies, Vol. 82, Princeton University Press, Princeton, 1975.

[4] A. Borobia and V. Mazurovskii, Nonsingular configurations of 7 lines of $\mathbf{R P}^{3}$, Research Note 41, Department of Mathematics, Uppsala University, 1995.

[5] H. Crapo and R. Penne, Chirality and the isotopy classification of skew lines in projective 3-space, Adv. in Math., 103(1) (1994), 1-106.

[6] Yu.V. Drobotukhina, An analogue of the Jones polynomial for links in $\mathbf{R P}^{3}$ and a generalization of the Kauffman-Murasugi theorem, Leningrad Math. J., 2(3) (1991), 613-630.

[7] R.H. Fox, Free differential calculus I, Annals of Math., 57 (1953), 547-560.

[8] _ Free differential calculus II, Annals of Math., 59 (1954), 196-210.

[9] V.F. Mazurovskii, Configurations of six skew lines, J. Soviet Math., 52(1) (1990), 2825-2832.

[10] H.R. Morton, Exchangeable Braids, London Math. Soc. Lecture Notes, Vol. 95, 86-105, R.A. Fenn, 1985.

[11] H.R. Morton, Threading knot diagrams, Math. Proc. Camb. Phil. Soc., 99 (1986), 247-260.

[12] R. Penne, Multi-variable Burau matrices and labeled line configurations, Journal of Knot Theory and Its Ramifications, 4(2) (1995), 235-262.

[13] D.W. Sumners and J.M. Woods, The monodromy of reducible plane curves, Invent. Math., 40 (1977), 107-141.

[14] G. Torres, On the Alexander polynomial, Annals of Math., 57(1) (1953), 57-89.

[15] V.G. Turaev, Reidemeister torsion in knot theory, Russian Math. Surveys, 41(1) (1986), 119-182.

[16] O.Ya. Viro, Topological problems concerning lines and points of three-dimensional space, Soviet Math. Dokl., 2(32) (1985), 528-531.

[17] O.Ya. Viro and Yu.V. Drobotukhina, Configurations of skew lines, Leningrad Math. J., 1(4) (1990), 1027-1050.

Received April 6, 1997 and revised August 10, 1997. The author is attached to the Department of Mathematics and Computer Science, University of Antwerp (UIA).

KAREL De Grote-Hogeschool

ANTWERP, BELGIUM

E-mail address: penne@uia.ua.ac.be 